*For correspondence: machne@hhu.de (RM); berni@bioinf.uni-leipzig.de (SHB); studla@bioinf.uni-leipzig.de (PFS); 7 ilka.axmann@hhu.de (IMA); mabawsa@gmail.com (DBM)

\title{
Spatio-temporal Coordination of
}

\section{Transcription During the Cell Growth Cycle in Budding Yeast.}

\author{
Rainer Machné ${ }^{1,2, *}$, Stephan H. Bernhart ${ }^{3}$, Ilka M. Axmann ${ }^{1}$, Peter F. Stadler ${ }^{3,4,5,6,7}$, \\ Dougie B. Murray ${ }^{8,9}$
}

${ }^{1}$ Institute for Synthetic Microbiology and; ${ }^{2}$ Institute for Quantitative and Theoretical Biology, Heinrich Heine University, D-40225 Düsseldorf, Germany; ${ }^{3}$ Bioinformatics Group, Department of Computer Science, Interdisciplinary Center for Bioinformatics, Universität Leipzig Härtelstraße 16-18, D-04107 Leipzig, Germany; ${ }^{4}$ Max Planck Institute for Mathematics in the Sciences, Inselstraße 22, D-04103 Leipzig, Germany; ${ }^{5}$ Institute for Theoretical Chemistry, University of Vienna, Währingerstraße 17, A-1090 Wien, Austria; ${ }^{6}$ Facultad de Ciencias, Universidad Nacional de Colombia, Sede Bogotá, Colombia; ${ }^{7}$ Santa

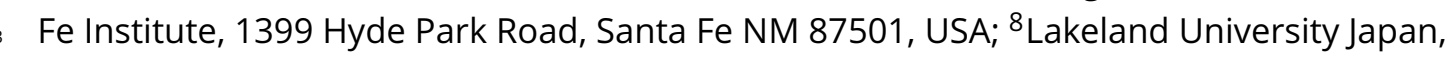
Shinjuku-ku, Tokyo 160-0022, Japan; ${ }^{9}$ University of Maryland Global Campus-Asia, Yokota Air Base, Fussa-shi, Tokyo 197-0001, Japan 18

\begin{abstract}
Metabolic self-synchronization in yeast cultures highlights significant gaps in our understanding of fundamental cellular processes. The oscillation percolates throughout cellular physiology, and the transcriptome oscillates between expression of genes encoding for biosynthesis and growth, and for catabolism and stress-response. However, long protein half-lives dampen this oscillation of transcript abundances, and putative functions remain elusive. We analyze an RNA-seq time series over 2.5 cycles of a short period respiratory oscillation $(\approx 36 \mathrm{~min}$, strain IFO 0233), preceding a distinct bifurcation of system dynamics. An integrative analysis of metabolic dynamics and co-expression cohorts identifies $\mathrm{CO}_{2}$ and carbonic anhydrase as putative players in oscillation and culture synchronization via a feedforward from catabolic to anabolic metabolism. Differential modulation of the relative duration of biosynthetic and catabolic protein cohort transcription precedes the bifurcation, compatible with a causative role in growth rate-dependent cellular resource allocation. The temporal program of protein-coding transcripts encodes the spatial structure of the cell. We suggest a function of periodic transcription in subcellular pattern formation of the growing cell.
\end{abstract}

\section{Introduction}

When yeast cultures are grown to a high cell density they tend to show collective metabolic dynamics. Numerous studies on continuously grown yeast have shown that stable oscillatory dynamics propagate throughout the metabolome, transcriptome and impinge on chromatin organization. The cycle period is dependent on growth conditions and the strain employed. However, under equivalent growth conditions cycle period can be categorized into long ( $\tau=4 \mathrm{~h}-5 \mathrm{~h}$ ) or short period cycles ( $\tau=0.7 \mathrm{~h}-1 \mathrm{~h}$; peculiar to the diploid strain IFO 0233). The cell division cycle (CDC) is tightly or loosely coupled with the long or short period cycles, respectively. The phenomenon is considered a model system for circadian cell biology in the absence of a circadian clock (Causton et al., 2015; 
Mellor, 2016), or more generally, for multi-scale coherence of cellular processes (Sonnleitner et al., 1997; Lloyd and Murray, 2007; Aon et al., 2008).

A key feature of long period oscillation that is often ignored or misinterpreted in current literature, is that the phase of low oxygen consumption (LOC) is not (respiro-)fermentative but purely respiratory. This is indicated by the respiratory quotient $(\mathrm{RQ})$, the ratio of carbon dioxide production and oxygen consumption, and $\mathrm{RQ}=\frac{q_{\mathrm{CO}_{2}}}{q_{\mathrm{O}_{2}}} \approx 1$ in LOC (Küenzi and Fiechter, 1969; von Meyenburg, 1969b; Strössle et al., 1988). LOC is a phase of volume growth and build-up of glycogen and trehalose at intermediate catabolic rates, in other words: the G1 phase of the CDC (Münch et al., 1992). The phase of high oxygen consumption (HOC) then marks the budding phase $(\mathrm{S}, \mathrm{G} 2)$, fueled by rapid mobilization of glycogen, leading to saturation of the respiratory capacity and a short phase of overflow metabolism with ethanol secretion (Sonnleitner and Käppeli, 1986; Sonnleitner et al., 1997; Futcher, 2006). DNA replication (S-phase) is initiated at maximal respiration rate (Burnetti et $a l_{.}, 2016$ ) and followed by the transition to LOC and cell division (Münch et al., 1992). The period is lower than the average culture doubling time due to the asymmetric nature of budding yeast division, and corresponds to the division time of mother cells. The smaller offspring cells (buds) traverse two or more cycles until large enough for division (Bellgardt, 1994a; Hjortso and Nielsen, 1995; Duboc and von Stockar, 2000).

However, periods longer or much shorter than the average culture doubling time can be observed in the same strains, depending on experimental conditions (von Meyenburg, 1969b; Heinzle et al., 1983; Porro et al., 1988; Bellgardt, 1994b). As Beuse et al. (1998) put it: "the scatter of the data contrasted with the convincing clarity of the theoretical model". The group of Kuriyama discovered stable short period oscillation in the strain IFO 0233 (Satroutdinov et al., 1992; Keulers et al., 1996). Here, HOC and LOC phases appear contracted and several processes are phase-shifted. Fermentation (RQ $>1$ ) and glycogen mobilization occur in LOC, RQ $<1$ in HOC, and no phase at $\mathrm{RQ} \approx 1$ is observed. Cell division is only partially synchronized, reflected by an initiation of S-phase in $8 \%-10 \%$ of cells at the transition from HOC to LOC phase (Klevecz et al., 2004; Sasidharan et al., 2012b). A biosynthetic pulse occurs at this transition, and many core carbon intermediates and amino acids peak in concentration (Murray et al., 2007). Population synchronization is lost at higher aeration rates, suggesting that gaseous substances act as synchronizers. Specifically, $\mathrm{CO}_{2}$ but not $\mathrm{O}_{2}$ (Keulers et al., 1996); $\mathrm{H}_{2} \mathrm{~S}$, a costly intermediate of the sulfate uptake pathway (Wolf et al., 2001; Murray et al., 2003); and the overflow metabolite acetaldehyde (Sohn et al., 2000; Murray et al., 2003) are key suspects.

Despite these differences, a similar temporal program of gene expression is observed in both short period (IFO 0233) and long period experimental systems (Machné and Murray, 2012). Both show maxima and minima of the cellular ATP/ADP ratio during the HOC and early LOC phases, respectively (von Meyenburg, 1969b; Satroutdinov et al., 1992; Xu et al., 2004; Müller, 2006; Machné and Murray, 2012; Amariei et al., 2014). Global remodeling of promoter and gene body nucleosome organization occurs during the late LOC phase (Amariei et al., 2014; Nocetti and Whitehouse, 2016). During HOC, transcription progresses from a ribosome biogenesis cohort (Ribi) and cytoplasmic ribosomal protein genes (RP), to amino acid synthesis genes (AA) and mitochondrial ribosomal protein genes (mtRP) at the transition to LOC. During the LOC phase, transcripts of a large group of stress-response and catabolic proteins (S/C) peak. In IFO 0233, the latter overlaps with expression of the mtRP cohort, while in long period systems these are separated (Machné and Murray, 2012). Several hypotheses on putative functions of the temporal program have been suggested. The functional profiles of co-expressed cohorts matches metabolic activity, and the initial hypothesis was a "just-in-time" model of gene expression (H1), where enzymes are expressed when required within the metabolic cycle (Klevecz et al., 2004; Tu et al., 2005; Murray et al., 2007). However, protein half-lives in yeast are now thought to be much shorter (Christiano et al., 2014) than initially reported (Belle et al., 2006). This would strongly dampen periodic transcription on protein level (Lück et al., 2014). Indeed recent proteomic studies found no (Feltham et al., 2019) 
or only few (O' Neill et al., 2020) periodic proteins in long period systems. Thus, Feltham et al. (2019) suggest a null hypothesis $(\mathrm{HO})$ where periodic mRNA production and degradation produce no significant costs and are a side-effect of the metabolic states the cells traverse. Slavov and Botstein (2011) and Burnetti et al. (2016) suggested an alternative hypothesis, based on the observation that the relative duration of the LOC phase varies strongly while HOC duration only subtly changes with growth rate. This would result in different absolute levels of the proteins from HOC-and LOC-specific co-expression cohorts and could underlie growth rate-dependent cellular resource allocation (Maaløe, 1979; Molenaar et al., 2009). Due to the obvious analogy we refer to this hypothesis as the "pulse-width-modulation" model (H2). More recently, O' Neill et al. (2020) suggested that a main function of the cycle lies in protein homeostasis, where ionic composition changes over the cycle and proteins aggregate and are degraded (autophagy) in LOC. Notably, these hypotheses are not mutually exclusive and each could contribute to a more comprehensive picture of this fascinating experimental system and of cell biology in general.

Despite numerous transcriptome studies in different strains and at different periods (Klevecz et al., 2004; Tu et al., 2005; Li and Klevecz, 2006; Slavov et al., 2011; Chin et al., 2012; Kuang et al., 2014; Wang et al., 2015), no analysis of the putative contributions of noncoding transcription during the cycle has yet been presented.

Thus, we performed strand-specific RNA-sequencing in high temporal resolution over 2.5 cycles of the short period oscillation of the strain IFO 0233 , and preceding a period-lengthening bifurcation of system dynamics. Global system variables, such as ATP flux and proton export, oscillated, confirming the cell-wide nature of the cycle. The protein coding transcriptome followed the conserved program but a pronounced shift from HOC-specific to LOC-specific transcript abundance peaks occurred within just two cycles. We systematically tested aforementioned hypotheses on putative functions of the temporal program of protein coding transcription, and report a pronounced enrichment of noncoding transcript abundance in LOC phase.

\section{Results and Discussion}

\section{Multi-generational Dynamics and a Bifurcation to Longer Period}

We studied short period oscillatory transcriptome dynamics in a continuous culture of the budding yeast strain IFO 0233. The culture traversed complex dynamic transients instead of the more commonly studied stable oscillation (Figure 1A and S4). The multi-generational transients spanned $30 \mathrm{~h}-40 \mathrm{~h}$ and appeared twice, with highly similar trajectories.

During these transients the oscillation followed the typical pattern observed for this strain and conditions, with period $\tau$ of $0.6 \mathrm{~h}-0.7 \mathrm{~h}$ (Figure 1B-C): the culture cycled between a phase of low oxygen consumption (LOC, here: $-q_{\mathrm{O}_{2}}<3.5 \mathrm{mmol} / \mathrm{h} / \mathrm{g}_{\mathrm{DCw}}$ ) with additional fermentation (respiratory quotient $\mathrm{RQ}=\frac{q_{\mathrm{CO}_{2}}}{-q_{\mathrm{O}_{2}}}>1$ ), and a phase of high oxygen consumption (HOC). During $\mathrm{HOC}$, RQ decreased below the $\frac{2}{3}$ stoichiometry of ethanol oxidation (Figure 1D). This is consistent with re-uptake of ethanol (Satroutdinov et alo, 1992) but could indicate further unknown contributions to $\mathrm{CO}_{2}$ turnover. Proton export ( $q_{\mathrm{H}^{+}}$, Figure $1 \mathrm{C}$ and E) peaked in early HOC phase, consistent with a higher intracellular pH during $\mathrm{HOC}$ (Keulers et al., 1996). The temporal mean $\overline{\mathrm{RQ}}>1$ (Figure 1A) indicates net fermentation during the dynamic state, but stoichiometric estimation of ATP production rates $q_{\text {ATP }}$ suggests that fermentation did not contribute significantly to overall ATP turnover (Figure 1D). The estimated ATP turnover rates are in phase with previously measured ATP/ADP ratio and energy charge, peaking in early to mid HOC phase (Machné and Murray, 2012; Amariei et al., 2014). The concentration of $\mathrm{H}_{2} \mathrm{~S}$ peaked at $\approx 3 \mu \mathrm{M}$ with a sharp increase upon transition to LOC (Figure 1C and $\mathrm{E}$ ), consistent with its release during amino acid biosynthesis in this transition phase (Murray et al., 2007) and its suggested role in population synchronization (Murray et al., 2003). During LOC, metabolic heat dissipation $\left(\Delta Q^{\prime}\right)$ fell to minima equal to that of the starving culture before onset of continuous feeding (Fig. S4).

Both multi-generational transients were characterized by gradual amplitude and period drifts. 

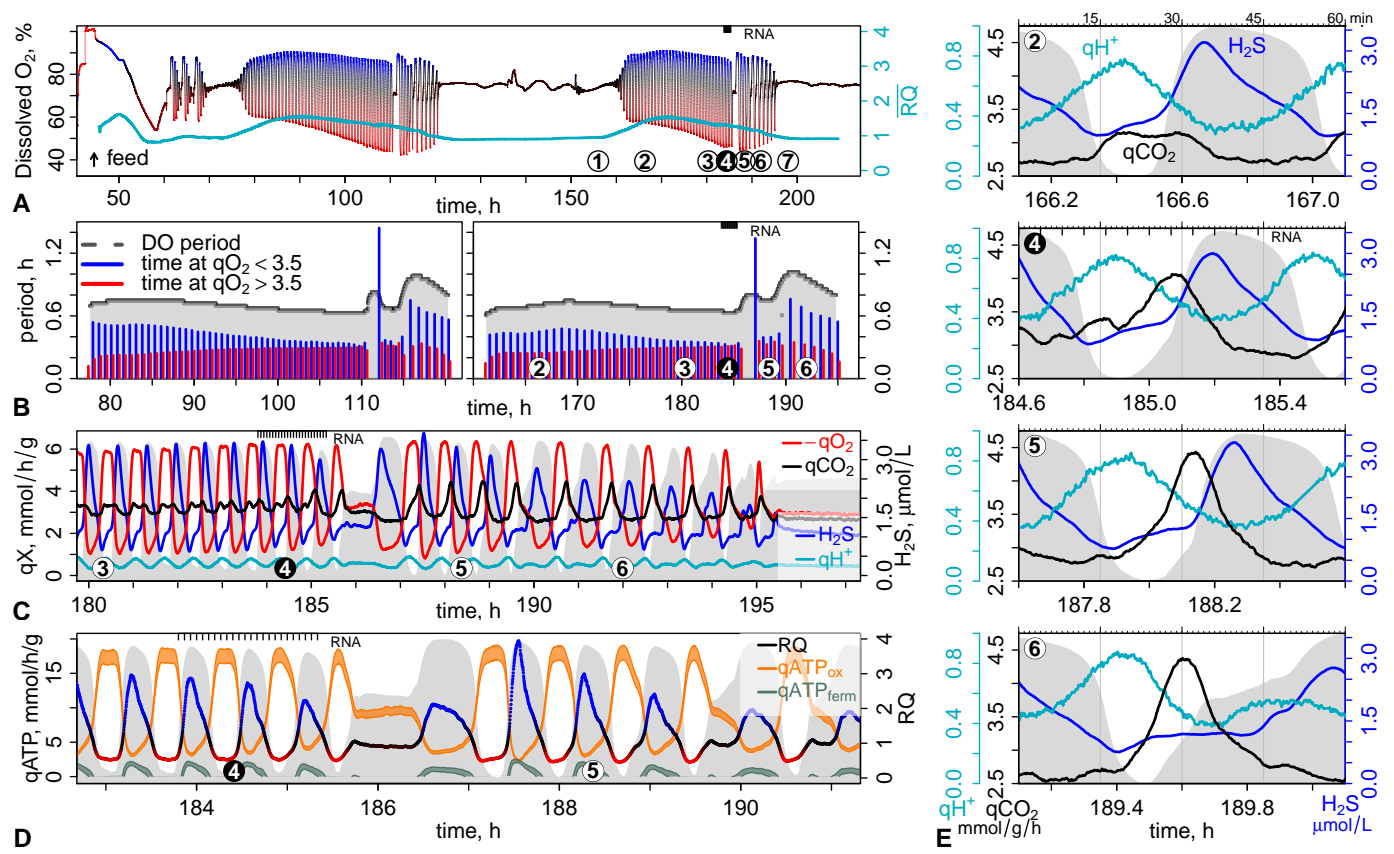

Figure 1. A Transient Oscillation \& the RNA-seq Experiment. A: Metabolic dynamics during continuous culture of the budding yeast strain IFO 0233. Dissolved $\mathrm{O}_{2}$ (DO) measurement from the start of continuous feeding (dilution rate $\phi=0.089 \mathrm{~h}^{-1}$ ). Line colors are derived from the respiratory quotient RQ (panel D) and indicate phases of high $\mathrm{O}_{2}$ consumption (HOC: red) and low $\mathrm{O}_{2}$ consumption (LOC: blue). The cyan line and right axis show the temporal mean $\overline{\mathrm{RQ}}$, a moving average over ca. $10 \mathrm{~h}$. Bullet points $\mathrm{P} 1-\mathrm{P} 7 \mathrm{on}$ the $\mathrm{x}$-axis are discussed in the text and used in (B-E). B: The oscillation periods for the dynamic transients were derived from a Wavelet transform of the DO signal and the phase lengths are the time spans of each cycle where oxygen uptake $\left(-q_{\mathrm{O}_{2}}\right)$ stayed below (red) or above (blue) $3.5 \mathrm{mmol} / \mathrm{h} / \mathrm{g}_{\mathrm{Dcw}}$. C: zoom on P3-P6 for measured metabolic rates and concentrations. The gray background indicates dissolved $\mathrm{O}_{2}$ as shown in $(\mathrm{A}) ; q_{\mathrm{O}_{2}}, q_{\mathrm{CO}_{2}}$ and $\mathrm{H}_{2} \mathrm{~S}$ were measured in the offgas of the reactor, corrected for the measurement delay and $\mathrm{H}_{2} \mathrm{~S}$ concentration was derived via its solubility. Proton export $\left(q_{\mathrm{H}^{+}}\right)$was calculated from the $\mathrm{NaOH}$ addition rate. D: zoom on P4-P5 for calculated rates. The respiratory quotient (RQ) and ATP production rates by respiration ( $q_{\text {ATPox }}$ ) or by fermentation ( $q_{\text {ATPferm }}$ ) were calculated from $q_{\mathrm{O}_{2}}$ and $q_{\mathrm{CO}_{2}}$, ranges of $q_{\text {ATP }}$ show values for pure glucose oxidation and fermentation, or pure fermentation of glucose and oxidation of only ethanol. The RQ color gradient serves as a reference in panel A and Figure S6. E: one-hour snapshots of typical dynamics at different phases of the transient (bullet points).

The overall period and LOC phase duration decreased while maximal oxygen uptake and HOC phase duration increased (bullet points P2-P3, Figure 1A, B, S4). As periods reached a minimum of $\tau \approx 0.6 \mathrm{~h}$, the plateau of $q_{\mathrm{CO}_{2}}$ was split into two distinct peaks flanking the HOC phase (P3-P4, Figure $1 \mathrm{C}$ and E). The first $q_{\mathrm{CO}_{2}}$ peak vanished and a sharper high amplitude peak remained at the transition to LOC, immediately preceding a distinct bifurcation of system dynamics. An additional phase first appeared at this bifurcation: metabolism was respiratory $(R Q \approx 1)$ and all parameters remained at intermediate values for about one full oscillation cycle (P4-P5, Figure 1C, D and S5). A delayed $\mathrm{H}_{2} \mathrm{~S}$ peak then preceded a long and pronounced LOC phase. The system then fell back to a shorter period, but $q_{\mathrm{CO}_{2}}$ remained phase-delayed and with higher amplitude (P5, Figure $1 \mathrm{C}$ and E). Finally, the period suddenly increased to $\tau \approx 1 \mathrm{~h}$, and the pause between HOC and LOC phases re-appeared as a short plateau or shoulder, again at $\mathrm{RQ} \approx 1$, and flanked by pulses of $\mathrm{CO}_{2}$ and $\mathrm{H}_{2} \mathrm{~S}$ release (P6, Figure 1C, E, S6).

In summary, we confirmed that the oscillation is a cell-wide phenomenon where global variables such as ATP flux, proton export or metabolic heat dissipation oscillate at high amplitude. Here, a gradual uncoupling of $\mathrm{CO}_{2}$ release from respiratory activity preceded a bifurcation of system dynamics to longer periods and a biphasic LOC. A new phase appeared at the transition from HOC to LOC, characterized by purely respiratory metabolism. Low but purely respiratory activity 
at $\mathrm{RQ} \approx 1$ is characteristic of the LOC phase in long period oscillations where glycogen is built up instead of degraded (Münch et al., 1992). In our experiment this phase was preceded by a novel pulse of $\mathrm{CO}_{2}$ release and the $\mathrm{H}_{2} \mathrm{~S}$ pulse was delayed and invariantly accompanied the transition to LOC. These observations are compatible with the suggested roles of both $\mathrm{CO}_{2}$ (Keulers et al., 1996) and $\mathrm{H}_{2} \mathrm{~S}$ (Murray et al., 2003) in population synchronization and suggest that they act via different metabolic or signaling targets. $\mathrm{H}_{2} \mathrm{~S}$ is released during sulfate uptake and amino acid synthesis at the transition to $\mathrm{LOC}$ (Murray et al., 2007). $\mathrm{CO}_{2}$ is increasingly recognized as a substrate of biosynthesis via carbonic anhydrase and bicarbonate $\mathrm{HCO}_{3}^{-}$, including gluconeogenesis (Aguilera et al., 2005; Martin et al., 2017). Tentatively, the bifurcation of system dynamics may result from an imbalance between the storage carbohydrate cycle and the biosynthetic pulse at the transition to LOC. The increase in period indicates a transition to a lower growth rate and/or a stronger coupling to the cell division cycle. A down-shift of dilution rate may have stabilized these dynamics.

\section{RNA-seq in High Temporal Resolution: Non-coding Expression In LOC Phase}

\section{Similarity-Based Segmentation of RNA-seq Time Series Data.}

Numerous time series of the protein-coding transcriptome have revealed a constant temporal program of transcription cohorts which is unfolded at strongly varying periods from $40 \mathrm{~min}$ to $7.5 \mathrm{~h}$ (Machné, 2017; Mellor, 2016). Neither the effect of transient states of the oscillation nor non-coding transcription have been studied yet. We thus took 24 samples for RNA-seq analysis in high temporal resolution ( $4 \mathrm{~min}$ ) over the last 2.5 cycles before the intermediate pause at $\mathrm{RQ} \approx 1$ ( $\mathrm{P} 4$ and top axis ticks in Figure 1). The strand-specific sequencing reads were mapped to the reference genome (strain S288C, R64-1-1), yielding reads for $76 \%$ of the genome (Fig. S8A). $17 \%$ of all genome positions oscillated at $p_{\text {DFT }}<0.05$ (Fig. S8B). The read-count phase distribution was bimodal with peaks in mid HOC and late LOC phases (Fig. S8C). We previously developed a similarity-based segmentation algorithm (Machné et al., 2017) to reduce read-count data to ca. 37,000 segments, each a putative individual transcript (Figure 2A, B and S8D). Of these, 6344 segments (17\%) oscillated ( $p_{\text {rain }}<0.05$, Figure $2 \mathrm{C} \& \mathrm{~S} 8 \mathrm{E}-\mathrm{F})$, confirming that a large fraction of the the transcriptome undergoes periodic changes in abundance.

Next, we analyzed the overlap of our segments with annotated genome features and previously published transcript data sets (Fig. S9). 4489 segments were classified as protein-coding transcripts (Figure 2D). These coding segments reproduced the previously established transcriptome program (Machné and Murray, 2012), while periodically expressed non-coding and antisense transcripts predominantly peaked in the LOC phase (Figure 2E).

\section{Clustering Analysis.}

Very short and lowly expressed segments ( $26 \mathrm{k}$ with $p_{\text {rain }} \geq 0.85$ ) were removed from further analyses, and the remaining $11 \mathrm{k}$ segments (Fig. S8G-I) were clustered into ten co-expressed cohorts. Clusters were sorted, re-labeled and colored by their mean peak phase (Fig. S10-S12). These ten cohorts can be further classified (Fig. S10C) into two groups of five clusters each. The high amplitude clusters (Figure 3 and S11) are characterized by long segments, and $61 \%-91 \%$ of segments assigned to a protein-coding gene. Low amplitude clusters (Figure 4 and S12) contain shorter and weakly expressed segments, with $21 \%-91 \%$ non-coding and spanning LOC phase. Thus, the clustering analysis confirms both observations: coding gene co-expression cohorts traverse the known temporal program while non-coding transcripts are predominantly expressed in LOC phase.

\section{A Conserved but Adaptable Temporal Program of Transcription}

The temporal sequence of transcript abundance peaks of the five protein-coding gene cohorts (Figure 3A: clusters 1-3, 7 \& 8) and their Gene Ontology enrichment profiles (Figure 3B and S13) recapitulate previous results (Klevecz et al., 2004; Machné and Murray, 2012): the RiBi regulon of ribosomal biogenesis genes (Jorgensen et allo, 2004) peaks in early to mid HOC phase (cluster 1: Ribi), followed by clusters encoding for cytoplasmic ribosomal proteins (cluster 2: cRP), and 

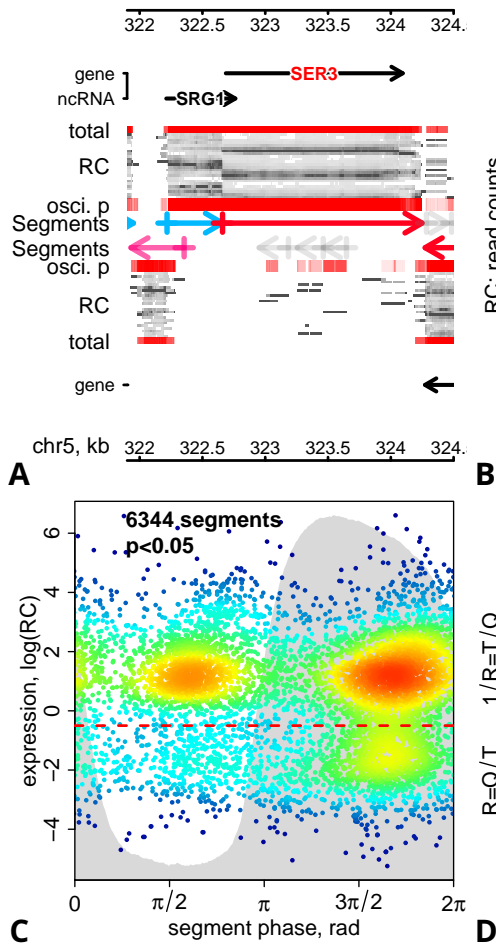
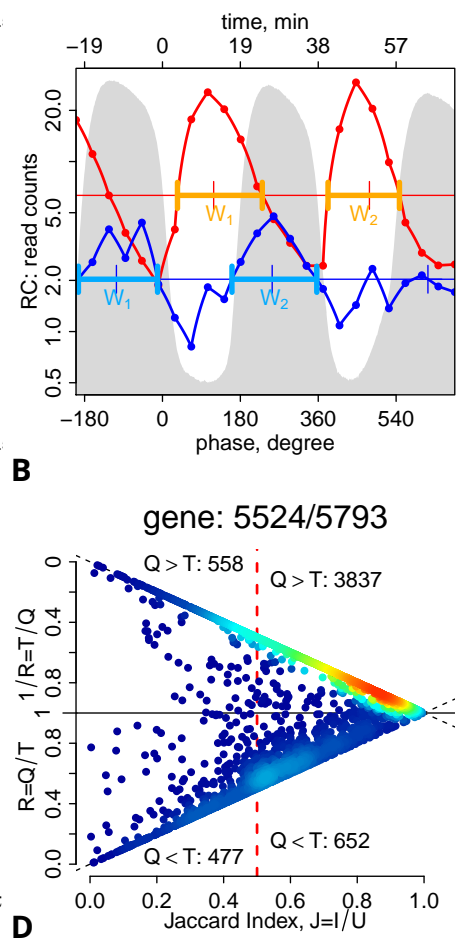

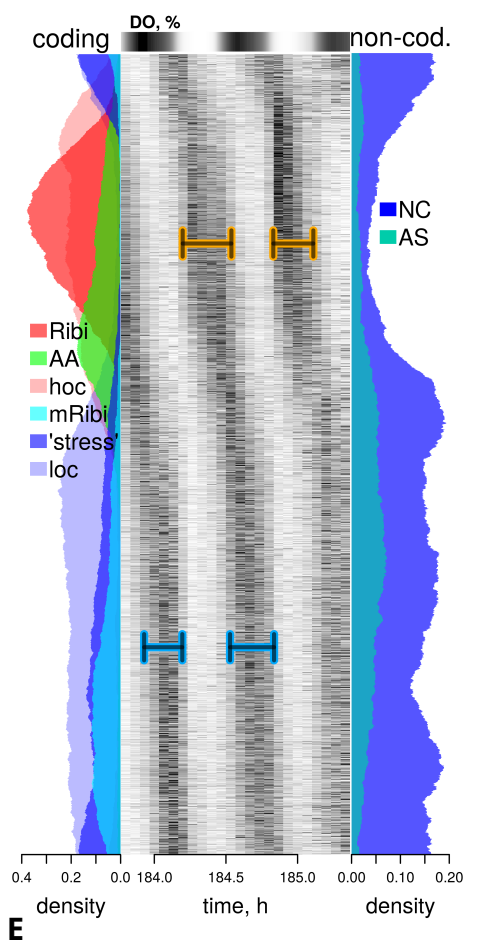

Figure 2. RNA-seq Data Structure and Segmentation. A: RNA-seq time series data at the SRG1/SER3 locus in a genomeBrowser plot. Forward strand data is shown in the top rows and reverse strand in the bottom rows in reverse order. From top to bottom for forward strand data: annotated genome features as arrows; "total" RNA-seq signal as red-scale heatmap (red: high); read-count time series ("RC", in RPM) from bottom to top as a gray-scale heatmap (dark: high); the oscillation p-value ("osci p.") as red-scale heatmap (red: low p-value); "Segments" as colored arrows. B: time series of the segments mapping to SRG1 (blue) and SER3 (red). The horizontal lines are the temporal medians, and the thicker bars indicate the peak widths $W$ in the first and second full cycle. C: mean read-counts (RC, in RPM) vs. oscillation phase of 6344 segments with $p_{\text {rain }}<0.05$ as a 2D histogram (red: high and blue: low local density). The gray background indicates the dissolved $\mathrm{O}_{2}$ concentration averaged over the the first two cycles of the experiment. The oscillation statistics (phase and p-value) were calculated only for the first cycles of the experiments. D: J-R plot (see Methods) of individual overlapping pairs of segments (query $Q$ ) with annotated coding genes (target $T$ ) as a 2D histogram (red: high and blue: low local density). $R$ is the length ratio and $J$ is the Jaccard index of an overlapping pair. The total number of overlapping and tested genes are indicated above the plot. The 4489 genes with $J>0.5$ where classified as coding gene transcripts in further analyses. E: phase-ordered heatmap of the time-courses of oscillatory segments (same as in (C)). The dissolved $\mathrm{O}_{2}(\mathrm{DO}, \%)$ is shown as a heatmap (black: high DO) on the top axis. Left and right panels show local densities (circular moving average of counts over $1 / 10$ of the total number) of segments overlapping with previously defined (Machné and Murray, 2012) classes of coding genes (left: Ribi, ribosomal biogenesis and cytoplasmic ribosomal proteins; AA: amino acid synthesis; mRibi: mitochondrial genes, incl. ribosomal proteins; stress: catabolic and protein homeostasis genes), or non-coding segments (right: AS, overlapping with an ORF in antisense; NC, no overlap in sense or antisense).

amino acid biosynthetic pathways (cluster 3: AA) at the transition to LOC. During the LOC phase, mitochondrial proteins, including mitochondrial ribosomal proteins (cluster 7: $\mathrm{mRP}$ ) are co-expressed with a stress response (Gasch et al., 2000; Brauer et al., 2005) or G1 phase (O'Duibhir et al., 2014) regulon, comprising proteins of the general stress response (chaperones) and of carbohydrate, fatty acid and protein catabolism (cluster 8: $\mathrm{S} / \mathrm{C}$ ).

\section{Transcriptome Meta-Analysis: Different Periods - Same Program.}

Similar observations were described in transcriptome analyses from different strains and conditions, with periods ranging from $40 \mathrm{~min}$ to $7.5 \mathrm{~h} \mathrm{(Li} \mathrm{and} \mathrm{Klevecz,} \mathrm{2006;} \mathrm{Tu} \mathrm{et} \mathrm{al.,} \mathrm{2005;} \mathrm{Chin} \mathrm{et} \mathrm{al.,} \mathrm{2012;}$ Kuang et al., 2014; Wang et al., 2015; Slavov et al., 2011). We used our clustering into five proteincoding co-expression cohorts to analyze seven data sets from five previous publications (Fig. S14- 
Scaled Expression
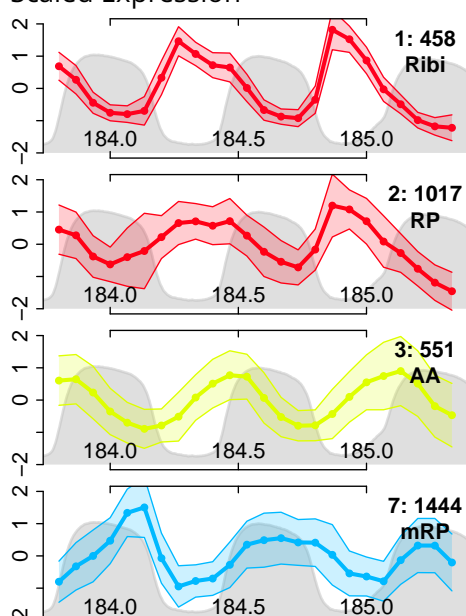

ฯ $184.0 \quad 184.5 \quad 185$

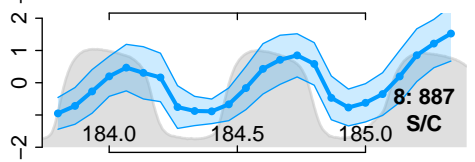

A

time, $\mathrm{h}$
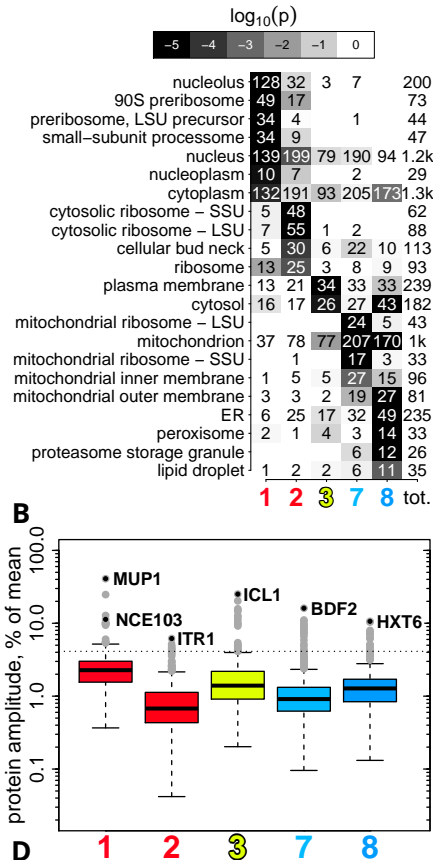

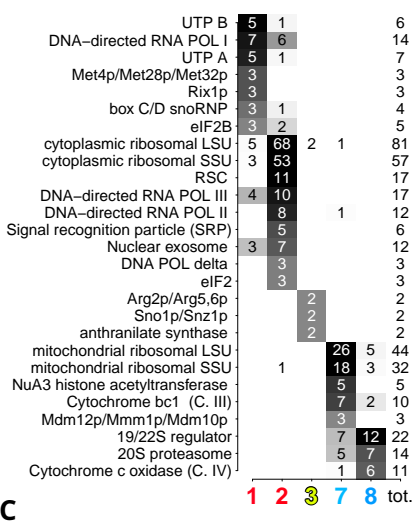

C

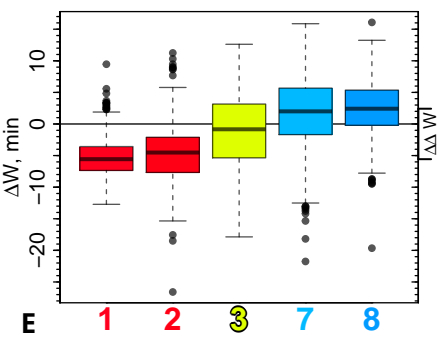

Figure 3. Segment Cluster Analysis. A: time series of the five major periodic co-expression cohorts. Segment time-courses (mean RPM) were normalized to mean of 0 and divided by their standard deviation ( $R$ function scale). The mean of each cluster is shown as a solid line with points indicating the sampling times, and standard deviations as transparent ranges; the legends indicate the cluster label, the number of segments in the cluster and the posterior functional cohort assignment. The gray background indicates the dissolved $\mathrm{O}_{2}$ (DO) concentration. B: Sorted cluster enrichment profiles for the Gene Ontology category "cellular component". The $p$-values were calculated by cumulative hypergeometric distribution tests and are shown as gray-scale. Only categories with $p<0.001$ (white text) and more then 10 genes in one of the clusters are shown. See Figure S13 for all clusters and GO categories. C: Cluster enrichment profile $\left(p_{\text {sort }}=0.01\right)$ of protein complexes in the CYC2008 annotation (Pu et al., 2009), colored as in (B). For a comprehensive picture of complex expression, the segment with the best overlap (highest Jaccard index $J>0$ ) was considered for each coding gene. See Figure S21 for all clusters. D: Box-plots of cluster distributions of predicted relative protein amplitudes $A_{\text {protein, }}$ estimated from transcript amplitudes and protein half-lives (Fig. S18). The horizontal line indicates the lowest $A_{\text {protein }} \approx 4 \%$ of the top 100 genes (Table S2). The top predicted oscillators of each cluster are indicated. E: Box-plots of cluster distributions of peak-width changes. $\Delta W=W_{2}-W_{1}$ is the difference of the peak widths (Figure 2B \& S20) between the second and first full expression cycle, and $\Delta \Delta W$ is the difference of $\Delta W$ medians of clusters 1 and 8.

S16). This meta-analysis emphasizes the universality and adaptability of the co-expression cohorts: firstly, a temporally constrained program $(0.5 \mathrm{~h}-2 \mathrm{~h})$ leads from Ribi/cRP via AA to mRP, ending with the transition from HOC to LOC (left panels in Fig. S15). Secondly, the HOC- and LOC-specific cohorts are always anti-correlated. In long period systems, the duration of $\mathrm{S} / \mathrm{C}$ expression increases with increasing duration of the LOC phase, while Ribi forms sharp peaks. Thirdly, the mRP and AA cohorts always peak at the transition from HOC to LOC but show variable timing between experiments (right panels in Fig. S15-S16). Interestingly, very similar temporal patterns can be observed in six distinct cell cycle arrest \& release experiments (Fig. S17) (Orlando et al., 2008; Bristow et al., 2014).

Next, we tested three hypotheses on putative functions of this universal yet adaptable "functiotemporal" expression program.

\section{$\mathrm{CO}_{2}$, Carbonic Anhydrase and the Glyoxylate Cycle: Novel Feedback Candidates.}

The temporal order of mRNA abundances makes intuitive sense as a just-in-time functional program (H1) coordinated with metabolic dynamics (von Meyenburg, 1969b; Klevecz et al., 2004; Tu et al., 2005; Murray et al., 2007; Machné and Murray, 2012). However, the effect of periodic mRNA on protein levels depends on protein half-lives. Thus, we estimated relative protein amplitudes from 
our RNA abundance profiles and protein half-life data (Christiano et al., 2014) using a mathematical model of periodic gene expression (Lück et al., 2014). The majority of proteins are predicted to vary by only $0.5 \%-1 \%$ of their mean (Figure 3D and S18). Only 21 proteins have relative protein amplitudes of $\geq 10 \%$, most from the Ribi and AA cohorts. The top 100 list of our predicted oscillators contains many cell wall proteins, nutrient transporters, transcription factors, and a few metabolic enzymes (Fig. S18, Tab. S3 and Dataset S3). Several proteins of the sulfate uptake pathway (MET genes) are expressed in Ribi, peaking prior to the pathway intermediate $\mathrm{H}_{2} \mathrm{~S}$ at the HOC/LOC transition (Figure 1). The carbonic anhydrase (NCE103, in Ribi) catalyzes the interconversion of carbon dioxide and bicarbonate $\left(\mathrm{CO}_{2}+\mathrm{H}_{2} \mathrm{O} \leftrightarrow \mathrm{HCO}_{3}^{-}+\mathrm{H}^{+}\right)$. The early downregulation of the Ribi cohort (Figure $3 \mathrm{~A}$ and $\mathrm{E}$ ) in the second sampled cycle correlates with the appearance of the $\mathrm{CO}_{2}$ pulse at transition to LOC (Figure 1E). In short period oscillations, population synchronization likely occurs via a gaseous substance, and evidence for both, $\mathrm{CO}_{2}$ and $\mathrm{H}_{2} \mathrm{~S}$, have been previously presented (Keulers et al., 1996; Murray et al., 2003). Both are substrates of biosynthetic metabolism (anabolism) and several key enzymes of biosynthesis are among our top predicted protein oscillators, notably three enzymes of the glyoxylate cycle (ICL1, CIT2, MDH2); all expressed with the AA cohort. In summary, several short-lived proteins with high RNA amplitudes are involved in the transition from energy generation (catabolism) during $\mathrm{HOC}$ to a biosynthetic pulse (anabolism) at the transition to LOC. In the Discussion, we will outline putative feedforward and feedback interactions behind this switch from HOC to LOC phase.

\section{Resource Allocation by Pulse-Width Modulation.}

The majority of proteins appear too stable to be affected by periodic transcript abundance. An alternative interpretation of the periodic transcription cohorts has been suggested (Brauer et al., 2005; Slavov and Botstein, 2011; Burnetti et al., 2016). Growth rate is directly proportional to the (cytoplasmic) ribosome fraction of the proteome, and the relative expression of different protein classes is adjusted to the given growth conditions (Maaløe, 1979; Molenaar et al., 2009). Variation of the relative times spent in HOC- and LOC-specific transcription states could serve to tune relative proteome fractions (H2). Shorter relative duration of the HOC phase and HOC-specific transcription would lead to a lower fraction of ribosome-associated proteins (Ribi and RP cohorts). Indeed, a pronounced shift to LOC-specific expression (S/C) with decreasing growth rate is seen in dilution rate shift experiments (Fig. S16D) (Chin et alı, 2012; Wang et alı, 2015). The heatmap (Figure 2E) and cluster average time-courses (Figure $3 \mathrm{~A}$ ) indicate a shift of relative times spent with HOC- or LOC-specific expression within just two cycles of the oscillation. To quantify this observation we calculated the peak widths $W_{1}$ and $W_{2}$ (Figure 2B \& S20) of the first and second fully traversed expression cycle and calculated peak width changes $\Delta W=W_{2}-W_{1}$. The S/C cohort gains on average about 8 min over the Ribi cohort ( $\Delta \Delta W$, Figure $3 \mathrm{E}), 22 \%$ of a full cycle, all within just two cycles of the oscillation. Thus, a pronounced shift to expression of the S/C cohort, indicative of a lengthened LOC and decreased growth rate, directly preceded the bifurcation in system dynamics to longer periods and a biphasic LOC (Figure 1).

\section{The Temporal Program Reflects the Spatial Structure.}

The resource allocation model can account for the observed relations to growth rate, and the model holds for different periods. However, it offers no interpretation of the temporal program, the fixed sequence of co-expression cohorts. Recent insights into the intricate spatio-temporal organization of protein homeostasis (Langdon et al., 2018; Shiber et al., 2018; Schwarz and Beck, 2019) offer a novel and complementary perspective. The Gene Ontology (GO) analysis by "cellular component" maps the temporal program onto the spatial organization of cell structures (Figure 3B): from nucleolus (Ribi), to nucleus (Ribi/RP), to cytosol, to plasma membrane (AA); followed by mitochondria (mRP), then large cytosolic structures, such as the endoplasmatic reticulum (ER), proteasomes and lipid droplets (S/C). Thus, the protein products of the temporally co-expressed genes also tend to co-localize at their final subcellular locations. Analysis of the CYC2008 protein 
complex annotation (Pu et al., 2009) confirms that many protein complexes are co-expressed within a cluster (Figure $3 C$ ). Large complexes ( $>10$ proteins) involved in basal cellular functions are co-expressed in either HOC or LOC, while complexes with regulatory function are comprised of differentially expressed subunits (Fig. S21B). In a growing cell, fresh ribosomes are constantly built and loaded with the current set of mRNA. A temporal expression program would lead to spatially structured layers of loaded ribosomes (H3) which can then be further sorted by specific peptide or mRNA motif-based mechanisms (Niessing et al., 2018).

We conjecture that the biosynthetic phase at the transition to LOC (Murray et al., 2007) may be directly followed by a global pulse of protein translation. This translation pulse would act on all cohorts, but their temporal expression sequence may have contributed to a spatial pre-ordering of ribosomes loaded with fresh mRNA cohorts. The subsequent low energy state during LOC could then be a phase of self-assembly of macromolecular complexes, including repair or removal of damaged complexes and protein aggregates ( $O^{\prime}$ Neill et al., 2020).

\section{Antisense and Non-Coding Transcription.}

Most antisense and non-coding transcript abundances peak in LOC phase (Figure 2E). How are non-coding transcripts related to the conserved temporal program of the coding transcriptome, and are they involved in its re-modeling at the bifurcation of system dynamics? Five low amplitude co-expression cohorts, clusters 4, 5, 6, 9 and 10 (Figure 4A), contain mostly non-coding transcripts (Fig. S12). Transcript abundance peaks of clusters 5, 6 and 9 span the LOC phase. Cluster 4 transcript abundances show an additional peak during the second $\mathrm{HOC}$ phase and a steep increase in the third LOC phase of our experiments. Cluster 10 peaks shift from late to early HOC phase.

\section{Antisense \& Promoter-Associated Transcripts: Response to Metabolic Transition.}

We analyzed the local neighborhood of segments and segment clusters. Non-coding RNA transcription can interfere with, and thereby regulate, coding genes in cis, either as antisense or as promoter-associated RNA (PAR) transcripts (Martens et al., 2004; Thompson and Parker, 2007; Servant et al., 2012; Nguyen et al., 2014; Ellison et al., 2019). Segment permutation tests show that all low amplitude clusters, except cluster 4, overlap with previously characterized classes of non-coding transcripts (Figure 4B, S22A). Antisense transcripts are less frequently found at Ribi and RP cohorts (Fig. S22B), but we find no systematic patterns between clusters (Fig. S22D). Various configurations of upstream, downstream and antisense transcripts can be observed at previously characterized antisense loci (Fig. S23). In contrast, clusters 4 and 10 are enriched within 500 bp upstream of protein-coding transcripts (Figure $4 \mathrm{C}$ ). Cluster 4 transcripts are enriched upstream of the RP, AA and $\mathrm{mRP}$ cohorts, and cluster 10 transcripts are enriched upstream of S/C. A direct comparison of the time-courses of such upstream:coding-pairs reveals an anti-correlation between expression of the upstream and downstream transcripts. During the last sampled cycle, abundances of cluster 4 PAR increase while their putative downstream targets decrease (Figure 4E). Cluster 10 PARs decrease as $\mathrm{S} / \mathrm{C}$ transcripts increase (Figure 4F). Inspection of individual loci (Figure 4E and F, and all 4:7 and 10:8 pairs in Fig. S24, S25) shows that cluster 4 segments often extend into the coding region. Cluster 10 transcripts are often found upstream of ORF transcripts and originate from tandem-oriented genes. Functional analysis of the target coding genes at the yeastmine webserver (Balakrishnan et al., 2012) shows enrichment for transcription regulation and DNA-binding (cluster 7), and for catabolic processes, ion membrane transport, oxidoreductase activity, plasma membrane-bound and mitochondrial membrane-bound proteins (cluster 8), respectively (Table S4).

\section{Complex Local Configurations and Genome Evolution.}

Visual inspection reveals highly individual configurations of upstream and antisense transcription that escaped the segmentation algorithm or cluster enrichment tests. For example, the seven alcohol dehydrogenase isozymes are associated with various pronounced noncoding antisense and PAR transcripts, all mostly in LOC phase (Fig. S26). ADH1 and ADH2 preferably catalyze formation 

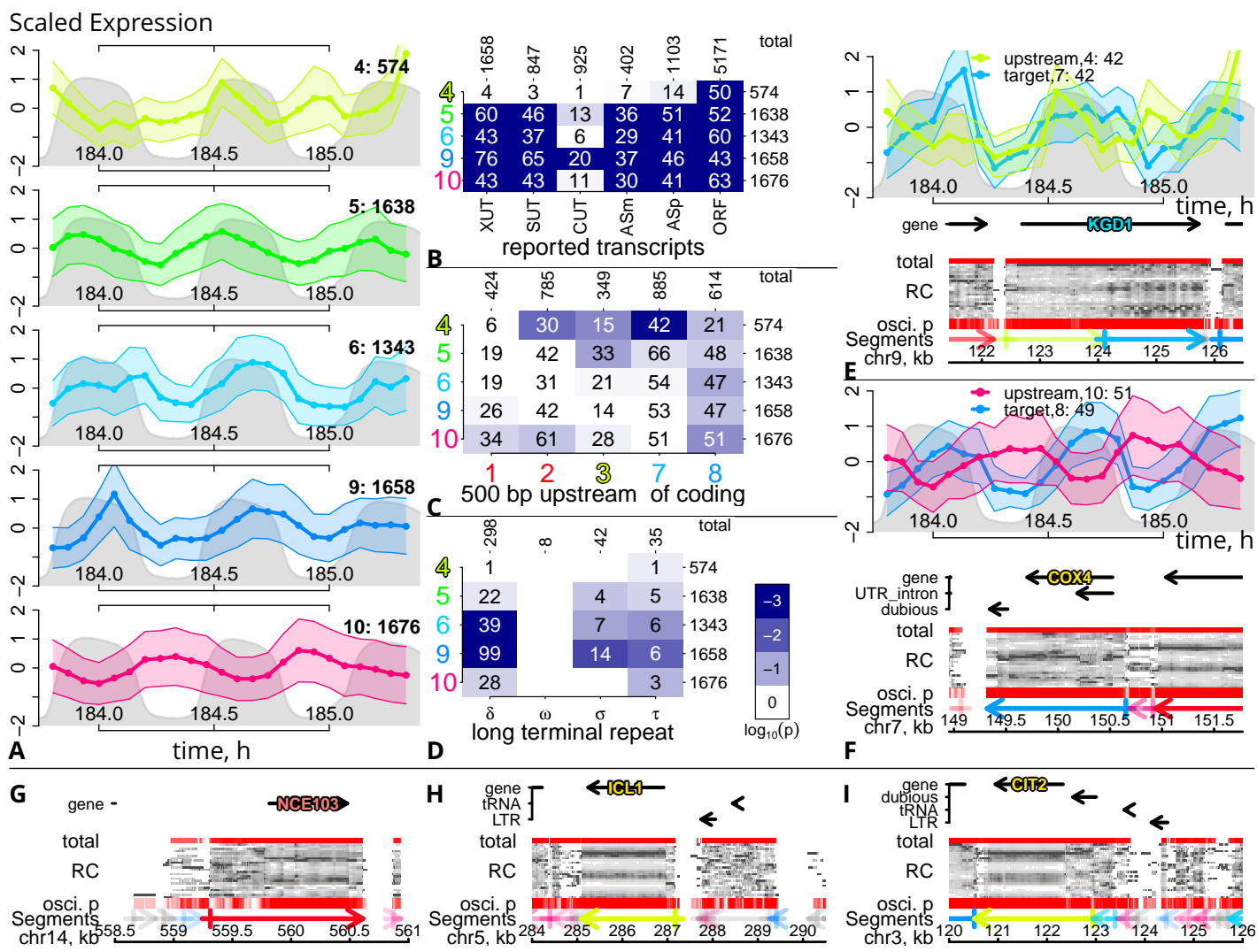

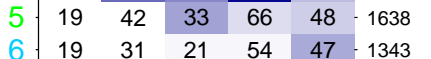

$\begin{array}{llllll}19 & 31 & 21 & 54 & 47 & -1343\end{array}$

$10 \begin{array}{llllll}34 & 61 & 28 & 51 & 51 & -1676\end{array}$

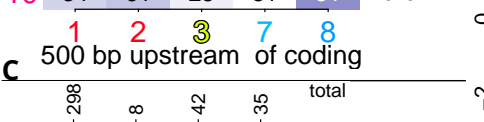

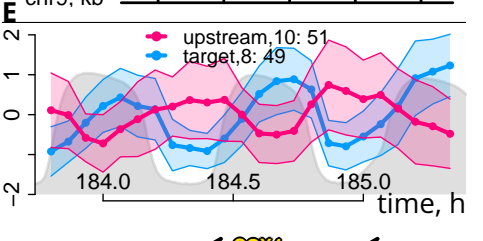

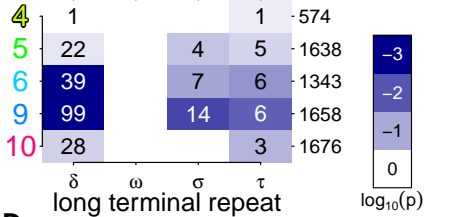

D long terminal repeat $\log _{10}(\mathrm{p}) \quad \mathbf{F}$

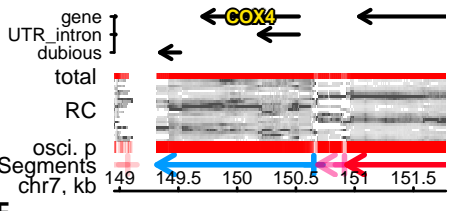

$\mathbf{F}$
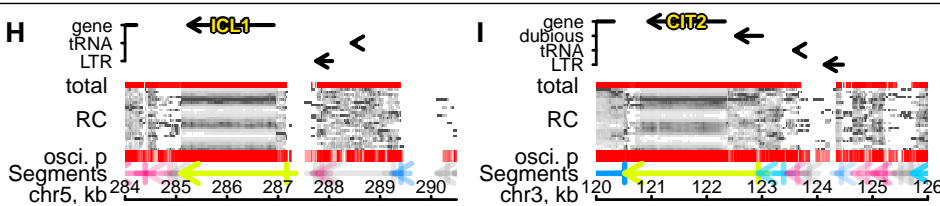

Figure 4. Non-coding clusters. A: time series for the cohorts 4, 5, 6, 9, 10, which comprise mostly segments without or little overlap with coding regions; plotted as described for Figure 3A. B-D: Cluster enrichment profiles of overlaps with experimentally characterized non-coding transcripts (B: SUT, CUT (Xu et al., 2009); XUT: (van Dijk et al., 2011); manually curated (ASm) and putative antisense transcripts (ASp): (Yassour et al., 2010)), with the 500 bp upstream of the 4489 coding segments (C), and with annotated long terminal repeats (D). Background and text colors indicate $p$-values from a segment overlap permutation test with $n=1000$ permutations (dark blue: $p \leq 0.001$; white text: $p \leq 0.05$ ). The rows (segments) were the permutated query set $Q$ in the enrichment tests. Numbers are the counts of overlapping segment pairs. Figure S22 provides results for all clusters. E: Time series of all 42 upstream/coding segment pairs from the middle panel of (B), with cluster 4 segments upstream of cluster 7 segments and the KGD1 gene as a typical example (Fig. S24 shows all examples). F: As (E) but for pairs with cluster 10 upstream of cluster 8 segments and example COX4 (Fig. S25 shows all, two downstream targets have two cluster 10 upstream transcripts). G-I: Promoter-proximal transcription at the predicted protein oscillators (Figure 3D) NCE103, ICL1 and CIT2.

and uptake of ethanol, respectively, and show opposite relations to an upstream transcript. The ADH2 ORF transcript and the ADH1 upstream transcript both increase in the last sampled cycle of the experiment, suggesting a coordinated regulation during the bifurcation of system dynamics. Various PAR and upstream transcripts are observed at HOC-specific anabolic genes, including the predicted high-amplitude protein oscillators CIT2, ICL1 and the carbonic anhydrase NCE103 (Figure $4 \mathrm{H}-\mathrm{I}$ and S27). These three genes and both isozymes of aconitase (ACO1, ACO2) are also flanked by long terminal repeats (LTR), recombination sites that flank LTR retrotransposons and often remained in the genome as solo-LTRs after loss of the transposon (Hani and Feldmann, 1998; Bleykasten-Grosshans et al., 2013). In each case, the LTRs are associated with short, noncoding transcripts from LOC clusters. Specifically, clusters 6 and 9 are enriched with annotated LTR elements (Figure 4D).

These observations highlight the wide diversity and complexity of transcription at each locus and emphasize that a broader chromosomal context needs to be considered for a more comprehensive understanding of non-coding transcription in LOC phase. 


\section{Conclusion}

Self-organized metabolic dynamics in yeast have been studied with basically constant culturing conditions since the seminal works of the Fiechter group on cell division cycle-coupled (long period) oscillations (Küenzi and Fiechter, 1969; von Meyenburg, 1969b,a; Futcher, 2006) and the Kuriyama group's discovery of the short period oscillation of the strain IFO 0233 (Satroutdinov et al., 1992; Keulers and Kuriyama, 1998; Sonnleitner et al., 1997; Lloyd and Murray, 2007). Specifically the latter system reveals time-scales of cellular self-organization below the cell division cycle.

Here, we characterized long multi-generational transients over $30 \mathrm{~h}-40 \mathrm{~h}$ in the IFO 0233 strain. Both, LOC duration and overall period became progressively shorter until a bifurcation to unstable oscillations at longer periods occurred (Figure 1, S6). We sampled for RNA-seq analysis at a minimum period, where both LOC and HOC phases took $0.3 \mathrm{~h}$, and shortly before the bifurcation. Within the 2.5 sampled cycles $(1.5 \mathrm{~h})$, transcript abundance peak widths and amplitudes shifted significantly (Figure 2E, Figure 3E). The cytoplasmic ribosome cohorts (Ribi/RP) were expressed for a shorter duration and the stress and catabolic cohort (S/C) was expressed for a longer duration. The mitochondrial cohort ( $\mathrm{mRP}$ ) was down-regulated and phase-advanced. And transcripts of the biosynthetic cohort (AA) diversified in both peak phase and peak duration. All of these relative expression changes were also observed in direct comparison between different previously published data sets, and specifically also in shifts to a lower growth rate (Fig. S14-S16). To understand these observations, we quantified the metabolic activity of the culture and systematically tested hypotheses on putative functions of periodic gene expression in the context of the transient dynamics. Figure 5 summarizes our main observations and interpretations.
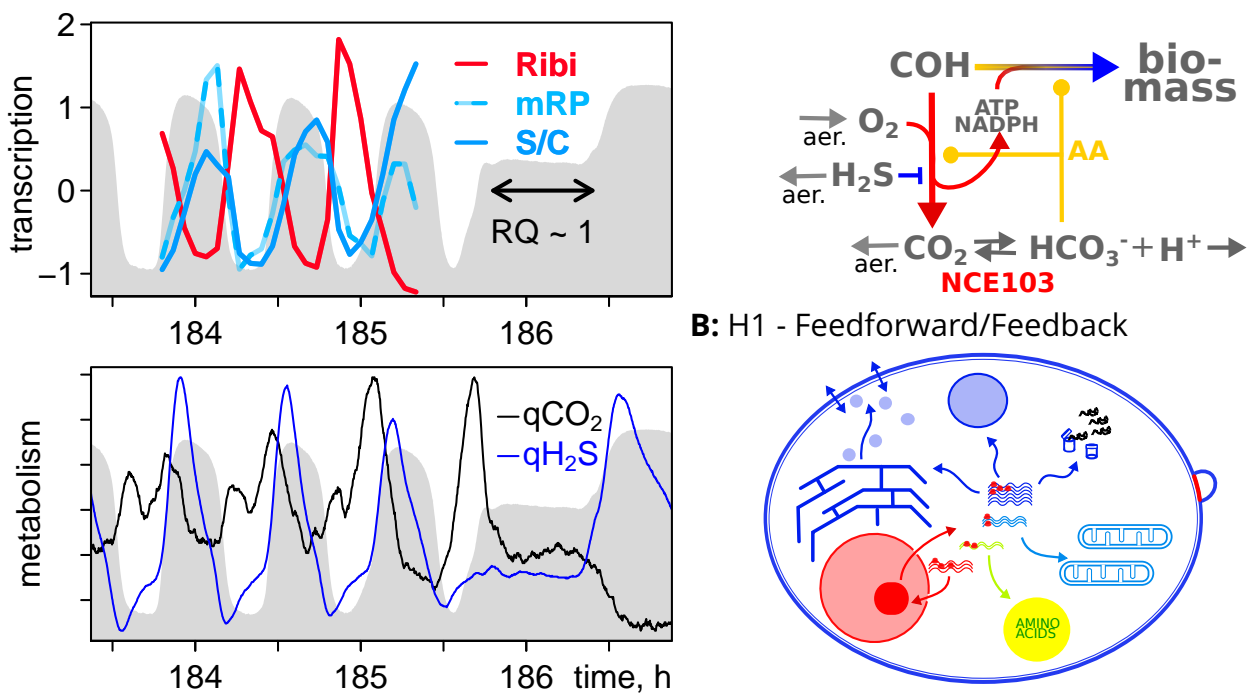

B: H1 - Feedforward/Feedback

A: Data Summary

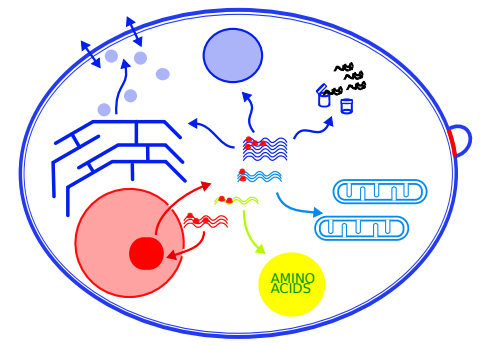

C: $\mathrm{H} 2+\mathrm{H} 3-\mathrm{PWM} \&$ Cell Structure

Figure 5. Data Summary \& Models. A: Summary of the main results. The top two panels show the mean normalized transcript abundances of the major co-expression cohorts (Figure 3 ), the bottom panels shows the $\mathrm{CO}_{2}$ and $\mathrm{H}_{2} \mathrm{~S}$ pulses at the transition to $\mathrm{HOC}$ phase (Figure 1). B: Putative feedback and feedforward interactions between catabolism and anabolism during the $\mathrm{HOC}$ to LOC transition mediated by $\mathrm{H}_{2} \mathrm{~S}$ and $\mathrm{CO}_{2}$ which may also participate in synchronization of the culture. Gaseous substances are lost through the aeration system (aer.). Oxidation of carbohydrates $(\mathrm{COH})$ occurs mostly in $\mathrm{HOC}$ phase (red arrow). A biosynthetic pulse (yellow/blue gradient arrow), catalyzed by enzymes from the AA cohort (yellow arrows), occurs at the transition to $\mathrm{LOC}$ phase, where several pathways require $\mathrm{HCO}_{3}^{-}$(A). C: The protein products of the co-expression cohorts are also co-localized in the cell, here indicated by the color scheme from Figure 3. Pulse-width modulation (PWM) of expression phases may adapt protein abundances to different growth conditions (Slavov and Botstein, 2011; Burnetti et al., 2016), and the temporal program could support spatial assembly of translating ribosomes as a first step of proteome sorting and transport. 
A Feedforward Gate at the HOC/LOC Transition.

At the transition from HOC to LOC a biosynthetic pulse occurs, where many amino acids and carbon intermediates reach their maximal concentration (Murray et al., 2007) as ATP is rapidly consumed (Machné and Murray, 2012; Amariei et alı, 2014). Respiro-fermentative metabolism and glycogen degradation are initiated (Satroutdinov et al., 1992), and 8\%-10\% of cells enter S-phase (Klevecz et al., 2004; Sasidharan et al., 2012b). In our experiment a new phase appeared at this transition, with respiratory metabolism $(R Q \approx 1)$ and at intermediate absolute rates; reminiscent of metabolic features of the LOC phase in long period systems, where glycogen is synthesized at RQ $\approx 1$ (Küenzi and Fiechter, 1969; Münch et al., 1992; O' Neill et al., 2020).

The $\mathrm{RQ} \approx 1$ phase was preceded by a novel pulse of $\mathrm{CO}_{2}$ release and followed by a phase-delayed $\mathrm{H}_{2} \mathrm{~S}$ pulse, which still marked the transition to LOC. Both gases have previously been suggested to participate in population synchronization (Keulers et al., 1996; Sohn et al., 2000; Murray et al., 2003). Several short-lived proteins with high mRNA amplitude from the Ribi and AA cohorts (Figure 3D and Tab. S2) are involved in their turnover and could thus mediate feedback between transcription and metabolism (H1, Figure $5 \mathrm{~B}) . \mathrm{H}_{2} \mathrm{~S}$ is a costly intermediate of the sulfate uptake pathway (MET genes in Ribi), which inhibits respiratory complex IV and could mediate a negative feedback from biosynthesis to oxidative catabolism (Wolf et al., 2001; Murray et al., 2007). Perhaps surprisingly, $\mathrm{CO}_{2}$ can be limiting in aerated cultures (Aguilera et al., 2005). Carbonic anhydrase (NCE103, Ribi) is required to shuffle $\mathrm{CO}_{2}$ to $\mathrm{HCO}_{3}^{-}$, which is a substrate of the first and/or ratelimiting reactions (Fig. S28) of nucleotide and fatty acid biosynthesis and for oxaloacetate synthesis by pyruvate carboxylase. The increase of intracellular pH (Keulers et al., 1996) and proton export during $\mathrm{HOC}$ (Figure 1E) would further support $\mathrm{CO}_{2}$ sequestration by NCE103. Similar observations, $\mathrm{pH}$ increase in $\mathrm{HOC}$ and protein abundance oscillation of NCE103, were recently reported from a long period oscillation in the CEN.PK113-7D strain ( $O^{\prime}$ Neill et al., 2020). The biosynthetic enzymes downstream of NCE103 are mostly expressed with the AA cohort which comprises several short-lived enzymes, e.g. those of the auto-catalytic glyoxylate cycle. In summary, these observations delineate a putative feedforward gate from catabolic to anabolic flux, where $\mathrm{CO}_{2}$ and ATP from respiration are both required to initiate the biosynthetic pulse (Figure 5B). In our experiment an imbalance between catabolic and anabolic flux may have blocked this gate and delayed the biosynthetic pulse. Such interactions would be in line with the just-in-time interpretation of the temporal expression program (H1), where transcription of short-lived enzymes can immediately affect metabolic dynamics.

\section{Pulse-Width Modulation and Subcellular Pattern Formation.}

However, given the long half-lives of most yeast proteins, periodic mRNA abundances should have only negligible effects on protein abundances (Figure 3D). Indeed, no or only few proteins were found to oscillate in abundance in two recent studies of long period systems (Feltham et allo, 2019; $O^{\prime}$ Neill et al., 2020). An alternative hypothesis (H2) has been suggested (Brauer et al., 2005; Slavov and Botstein, 2011; Burnetti et al., 2016). The co-expression cohorts peak preferentially during specific metabolic states, such as HOC, LOC or the transition phase. If a given phase lasts longer, e.g. due to some metabolic imbalance, more of the phase-specific genes will be translated to proteins. In this way, discrete on/off states of gene expression could be translated to continuous variation of the abundances of their protein products. Metaphors from electrical engineering appear apt: the oscillation could act as a digital-to-analog converter by pulse-width-modulation (PWM). Slow growing cells spend a lower fraction of the cycle in HOC (Fig. S16) (Slavov et al., 2014; Burnetti et al., 2016) which should lead to lower relative abundance of proteins from the ribosomal cohorts. Thus the PWM model of periodic gene transcription qualifies as a putative mechanism behind the old observation of a correlation between growth-rate and macromolecular cell composition (Maraløe, 1979; Molenaar et al., 2009).

During the multi-generational transients we observed a decreasing total cycle period and decreasing duration of LOC. According to the PWM model, this should have resulted in a slow increase of the ribosomal proteome fraction. Towards the bifurcation of system dynamics to longer 
periods this putative trend was reversed. Peak widths and amplitudes of the Ribi/RP, the mRP and $\mathrm{S} / \mathrm{C}$ cohorts all changed differentially within just 2.5 cycles (Figure $5 \mathrm{~A}$ ). Ribi/RP peak widths and mRP amplitudes decrease, while $\mathrm{S} / \mathrm{C}$ transcript abundances increase in both peak width and amplitude. After this remodeling of the transcriptome, the period and LOC duration were longer. A down-shift of dilution rate may have stabilized the oscillation at this longer period and lower growth rate. Our purely correlational data and the short snap-shot of the transcriptome do not allow definitive conclusions, but these relative shifts of the major co-expression cohorts are fully compatible with H2. Furthermore, we noted that the cohorts are strongly enriched for cellular co-localization of their products and for large protein complexes (Figure 3B, C, Figure 5C). It is increasingly recognized that many protein complexes assemble co-translationally, and artificial separation of subunit translation in space or time results in misfolding (Shiber et al., 2018; Schwarz and Beck, 2019). The temporal co-expression could ensure spatial pre-sorting of freshly loaded ribosomes in growing cells (H3), as a basis for more specific sorting mechanisms via mRNA- and peptide-motifs (Niessing et al., 2018). This interpretation is an extension of the PWM model and is perfectly compatible with the recently suggested role of the cycle in protein homeostasis ( $O^{\prime}$ Neill et al., 2020).

\section{Outlook: A Cell Growth Cycle?}

Periodic gene expression underlies several pattern formation phenomena in multi-cellular organisms (Moreno-Risueno and Benfey, 2011); in yeast it may help to structure colonies (Palkova et al., 2002). Here, we suggest that the temporal gene expression program underpins pattern formation even within the growing cell. In this integrative perspective, the cycle would reflect a fundamentally pulse-like growth behavior, where at each HOC to LOC transition a protein translation pulse, reflected in increased uptake of the nitrogen source (von Meyenburg, 1969b), generates new structured layers in the growing cell. Cell density increased in single yeast cells just before the G1/S transition, likely due to a pulse of protein translation (Bryan et al., 2010). Multiple growth pulses throughout the cell cycle have recently been observed in mammalian single cell studies (Liu et al., 2020), emphasizing the potential generality of such a model of cell growth. The LOC phase would be a low-energy macromolecular self-assembly phase where damaged cell structures and misfolded proteins are sequestered and degraded, potentially supported by increased protein aggregation (O' Neill et al., 2020) at low ATP (Mandl et al., 1952; Patel et al., 2017). Overall such a cell growth cycle would act as a global cell structural proofreading loop.

Finally, we observed a large diversity of differentially transcribed promoter-associated transcripts, but with no generalizable phase relations to their downstream coding genes. Locally individual configurations dominate the picture but we suggested a few examples, enriched at the LOCspecific coding cohorts (Figure 4E, F, S24, S25), for further experimental investigation of promoterassociated transcriptional interference dynamics. Antisense and non-coding transcript abundances were largely biased to LOC phase. Pervasive and weakly defined non-coding and LOC-specific transcription loci were enriched at Long Terminal Repeats of former retro-transposition events. These elements shape the evolution of the eukaryotic genomes (McClintock, 1950; Curcio et al., 2015; Moller et al., 2015). The chromosomal organization of the temporal expression program and the contributions of non-coding transcription in LOC phase will be analyzed in more detail in a subsequent publication.

\section{Materials and Methods}

\section{Strain History}

Kuriyama's lab first reported oscillations in continuous culture of the Saccharomyces cerevisiae strain IFO 0233 (Satroutdinov et al., 1992). The strain number is from the Japanese culture collection NBRC and is identified there as "Distillery yeast Rasse II", "accepted" in 1941, and as ATCC 560 in the US American culture collection. These strains can be traced back to the "Brennereihefe, Rasse II" isolated as "Hefe 128" axenic culture by Paul Lindner at the Berlin Institut für Gärungsgewerbe in 1889 from samples of a distillery in Gronowo (West Prussia, now Poland) which obtained their yeast 
from a dry yeast supplier in the city Thorn (now Torun, Poland) (Lindner, 1895). The strain and its descendant "Rasse XII" became commercially successful distillery strains within hybrid formulations ("Rasse $\mathrm{M}^{\prime \prime}$ ), and was at the time an intensively studied strain in basic research, e.g., in the search for the nature of "bios" (Lindner, 1919).

\section{Continuous Culture}

Pre-Culture

Saccharomyces cerevisiae (strain IFO 0233) were maintained on yeast nitrogen base agar plates (2\% glucose, $1.5 \%$ agar; Difco, Japan) at $4{ }^{\circ} \mathrm{C}$, sub-cultured from frozen stock cultures $\left(-80^{\circ} \mathrm{C} ; 1 \mathrm{~mL} ; 15 \%\right.$ glycerol; $5 \times 10^{8}$ cells). Pre-cultures were inoculated into Yeast Extract Peptone Dextrose media $\left(10 \mathrm{~mL} ; 1 \%\right.$ yeast extract, $2 \%$ peptone, $2 \%$ glucose) and grown at $30^{\circ} \mathrm{C}$ in an orbital incubator $(200 \mathrm{rpm})$ for $24 \mathrm{~h}$.

\section{Continuous Culture Medium \& Inoculation}

The culture medium consisted of D-glucose $\left(20 \mathrm{~g} \mathrm{~L}^{-1}\right),(\mathrm{NH} 4) 2 \mathrm{SO} 4\left(5 \mathrm{~g} \mathrm{~L}^{-1}\right), \mathrm{KH} 2 \mathrm{PO} 4\left(2 \mathrm{~g} \mathrm{~L}^{-1}\right), \mathrm{MgSO} 4.7 \mathrm{H} 2 \mathrm{O}$ $\left(0.5 \mathrm{~g} \mathrm{~L}^{-1}\right), \mathrm{CaCl} 2.2 \mathrm{H} 2 \mathrm{O}\left(0.1 \mathrm{~g} \mathrm{~L}^{-1}\right), \mathrm{FeSO} 4.7 \mathrm{H} 2 \mathrm{O}\left(20 \mathrm{mg} \mathrm{L}^{-1}\right), \mathrm{ZnSO} 4.7 \mathrm{H} 2 \mathrm{O}\left(10 \mathrm{mg} \mathrm{L}^{-1}\right), \mathrm{CuSO} 4.5 \mathrm{H} 2 \mathrm{O}$ $\left(5 \mathrm{mg} \mathrm{L}^{-1}\right), \mathrm{MnCl} 2.4 \mathrm{H} 2 \mathrm{O}\left(1 \mathrm{mg} \mathrm{L}^{-1}\right), 70 \% \mathrm{H} 2 \mathrm{SO} 4,\left(1 \mathrm{~mL} \mathrm{~L}^{-1}\right)$, Difco yeast extract $\left(1 \mathrm{~g} \mathrm{~L}^{-1}\right)$ and Sigma Antifoam A $\left(0.2 \mathrm{mLL}^{-1}\right)$. All chemicals were supplied by Wako Pure Chemical Industries Ltd., Japan. The medium prepared with this recipe has a $\mathrm{pH}$ of ca. 2.5 which allows for autoclaving of media with both sugar and ammonium without browning (caramelization) and further avoids precipitation of salts in feed medium bottles during continuous culture.

A custom-built bioreactor as outlined below was filled with $0.635 \mathrm{~L}$ of medium and autoclaved $\left(121^{\circ} \mathrm{C} ; 15 \mathrm{~min}\right)$. Aeration $\left(0.15 \mathrm{~L} \mathrm{~min}^{-1}\right)$, agitation $(750 \mathrm{rpm})$, and temperature $\left(30^{\circ} \mathrm{C}\right)$ and $\mathrm{pH}(3.4)$ control were switched on, until the system was equilibrated. Then, the dissolved oxygen probe was 2-point calibrated by flushing with pure nitrogen (0\%) and switching back to air (100\%).

The equilibrated and fully calibrated reactor was inoculated with $\approx 1 \times 10^{9}$ pre-culture yeast cells. A batch phase continued for $\approx 40 \mathrm{~h}$ until the cells had reached stationary phase, indicated by a sharp decrease in respiratory activity. Then continuous culture, i.e., feeding with fresh medium, was initiated (at $44.5 \mathrm{~h}$ in Figure 1).

\section{Culture Control \& Monitoring}

Continuous culture was performed in a custom-built bioreactor. The culture vessel was a jar fermentor (Eyela, Japan) with a total volume of $2.667 \mathrm{~L}$. Culture volume was measured using a balance (SB16001, Mettler Toledo, Japan), and continuous dilution with fresh medium was performed using a peristaltic pump (AC2110, ATTA, Japan) with a six roller planetary design which minimizes pulsing during rotation (about $10 \mathrm{rpm}$ ), and medium was pumped through $1 \mathrm{~mm}$ tubing (inner diameter; Masterflex, Cole Palmer, USA) and a 23 gauge steel needle. This ensured that the media was introduced in a stream of $<20 \mu \mathrm{L}$ droplets and just under a droplet per second at the operating dilution rate. Feed medium bottle weight was monitored by a balance (PMK-16, Mettler Toledo, Japan), set up to read from unstable environments and shielded from direct breezes.

The culture was agitated at $750 \mathrm{rpm}$ and aerated at $0.150 \mathrm{~L} \mathrm{~min}^{-1}$ by a mass flow controller (B.E. Marubishi, Japan). Dissolved oxygen was measured using an InPro 6800 sensor and pH with an InPro 3030 (both: Mettler Toledo, Japan).

Culture $\mathrm{pH}$ was maintained at 3.4 by the automatic addition of $2.5 \mathrm{~mol} \mathrm{~L}^{-1} \mathrm{NaOH}$, and the weight of the $\mathrm{NaOH}$ bottle was monitored on a balance (PM400). Local control of agitation and $\mathrm{pH}$ was carried out by Labo controllers (B.E. Marubishi, Japan). The reactor pressure was monitored by a manometer (DM-760, Comfix, Japan) installed on a split outlet flow stream. The culture temperature was controlled at $30^{\circ} \mathrm{C}$ by an external sensor connected to a circulating water bath (F25-ME, Julabo, Japan).

Partial pressure of oxygen and carbon dioxide in the off-gas were measured by an Enoki-III gas analyzer (Figaro engineering, Japan). The partial pressure of hydrogen sulfide in the off-gas was 
measured using an electrode based gas monitor (HSC-1050HL, GASTEC, Japan). Instruments were calibrated as per manufacturer's instruction.

\section{Reactor Data Acquisition and Calculation of Metabolic Rates}

Data were acquired via the in-house FERMtastic software at $0.1 \mathrm{~Hz}$. Metabolic rates were calculated as described previously (von Meyenburg, 1969a; Heinzle, 1987; Verduyn et al., 1991; Marison et al., 1998; Murray et al., 2007) from the online recorded data. Details and all equations are provided in Appendix A of the supporting information. All data were processed in the script samplingSeq_2019.R of the yeastSeq2016 git repository. All calculated rates are provided in Dataset S1.

\section{RNA Sequencing \& Read Mapping}

\section{Sampling, RNA Extraction \& Sequencing Library Generation}

Total RNA was extracted as previously described (Sasidharan et al., 2012a) from 24 samples taken every 4 min, covering ca. 2.5 cycles of the respiratory oscillation.

Culture samples were immediately quenched in ethanol and disrupted using acid-washed zirconia/silica beads ( $0.5 \mathrm{~mm}$; Tomy Seiko Co., Ltd., Japan) with sodium acetate buffer ( $250 \mu \mathrm{L}$; sodium acetate $300 \mathrm{mM}, \mathrm{Na2}$-EDTA $10 \mathrm{mM}, \mathrm{pH} 4.5-5.0$ ) and one volume of TE-saturated phenol (Nacalai Tesque) equilibrated with sodium acetate buffer $(250 \mu \mathrm{L})$.

The samples were then centrifuged $\left(12000 \mathrm{~g}, 15 \mathrm{~min}, 4^{\circ} \mathrm{C}\right)$ and the aqueous phase transferred to fresh $1.5 \mathrm{~mL}$ microcentrifuge tubes. Back-extraction was performed by adding sodium acetate buffer $(125 \mu \mathrm{L})$ to the bead-beat tubes, vortex $(10 \mathrm{~s})$, centrifuging $\left(12000 \mathrm{~g}, 15 \mathrm{~min}, 4^{\circ} \mathrm{C}\right)$ and adding the aqueous phase to the first aqueous phase. 2.5 volumes ice-cold $99.5 \%$ ethanol were added to the aqueous phase and RNA/DNA precipitated at $-20^{\circ} \mathrm{C}$ overnight. The samples were then centrifuged $\left(12000 \mathrm{~g}, 30 \mathrm{~min}, 4^{\circ} \mathrm{C}\right)$, the supernatant removed by aspiration, and pellets washed $3 x$ in $500 \mu \mathrm{L} 70 \%$ ethanol and air-dried (10 min, room temperature). DNA was removed (RNase-Free DNase Set; Qiagen, Japan) and RNA recovered by column purification (QIAquick PCR Purification Kit; Qiagen, Japan) in $50 \mu \mathrm{L}$ UltraPure water, and stored at $-80^{\circ} \mathrm{C}$ prior to analysis. Total RNA had an RNA integrity number $>7$ and $260 \mathrm{~nm}: 230 \mathrm{~nm}$ and $260 \mathrm{~nm}: 230 \mathrm{~nm}$ ratios $>2.14$. All cDNA libraries were then generated and sequenced by the Beijing Genome Institute (BGI), China. Strand specific cDNA libraries were created using the "dUTP method" (Parkhomchuk et al., 2009; Levin et al., 2010) and sequencing was carried out on an Illumina $1 \mathrm{G}$ sequencer.

\section{RNA-seq Read Mapping}

RNA-seq reads were mapped against the yeast reference genome (strain S288C, release R64-1-1) using segemehl (version 0.1.4) (Hoffmann et al., 2014) with default parameters and spliced read mapping enabled. Initially unmatched reads were mapped again using the remapping tool from the segemehl package and the resulting files were merged. Coverage (read-counts per nucleotide) was normalized for total library size to reads-per-million (RPM) and RPM values were stored in a bedgraph file for further analysis.

\section{RNA-seq Time Series Analysis}

Analysis Strategy and R Packages

All analyses were performed with bash and $\mathrm{R}$. The full analysis pipeline is available in a git repository at https://gitlab.com/raim/yeastSeq2016. Analysis and plotting tools developed for this work are available in an git repository with scripts and an R package available at https://github.com/raim/ segmenTools. RNA-seq segmentation was performed with the segmenTier R package (Machné et al., 2017), available at https://cran.r-project.org/package=segmenTier. Scripts for genome-wide data collections and mapping to the yeast S288C reference genome (release R64-1-1) as well as the genomeBrowser plots are available at the git repository https://gitlab.com/raim/genomeBrowser. 


\section{Additional Data Sources}

Genome annotations including Gene Ontology terms were taken directly from the gff genome file from the Saccharomyces genome database (SGD, release R64-1-1, 2011-02-08, same as for RNA-seq mapping). Published transcript data sets (XUT, SUT, etc.) were also obtained from SGD for the same genome release. Protein complex annotation CYC2008 (Pu et al., 2009) was downloaded from http://wodaklab.org/cyc2008/resources/CYC2008_complex.tab on 2019-06-04.

\section{Discrete Fourier Transform.}

A time series of $N$ measurements $x=\left\{x_{0}, \ldots, x_{N-1}\right\}$, taken at equally spaced time points $\left\{t_{0}, \ldots, t_{N-1}\right\}$, can be transformed to frequency-space by the Discrete Fourier Transform (DFT):

$$
X_{k}=\sum_{n=0}^{N-1} x_{n} e^{-2 \pi i \frac{k n}{N}} \quad, \quad k=\{0, \ldots, N-1\}
$$

where $X_{k}$ is a vector of complex numbers representing the decomposition of the original time series into a constant (mean) component (at $k=0$ ) and a series of harmonic oscillations around this mean with periods $P_{k}$, amplitudes $A_{k}$ and phase angles $\phi_{k}$ :

$$
\begin{aligned}
P_{k} & =\left(t_{N-1}-t_{0}\right) / k, \\
A_{k} & =\left|X_{k}\right| / N, \\
\phi_{k} & =-\operatorname{atan} 2\left(\operatorname{Im}\left(X_{k}\right), \operatorname{Re}\left(X_{k}\right)\right) .
\end{aligned}
$$

All DFT were performed with R's $f f t$ function.

For DFT-based clustering and segmentation analysis, it proved useful to scale DFT components by the mean amplitude of all other components $k>0$ :

$$
X_{k>0}^{\prime}=\frac{X_{k>0}}{\mid \overline{|X|}},
$$

and the constant component $(k=0)$ by the arcsinh transformation:

$$
X_{0}^{\prime}=\ln \left(\left|X_{0}\right|+\sqrt{X_{0}^{2}+1}\right)
$$

For analysis of read-count data $x_{n}$ were the raw read-counts, for analysis of segments $x_{n}$ were the mean of all read-counts of the segment.

The index $k$ corresponds to the number of full cycles with period $P_{k}$ in the time series. Only the first 19 time points, covering two full cycles of the oscillation were used for the calculation of phases and p-values, such that $k=2$ reflects the main oscillation. For all plots, phases were shifted such that $\phi_{2}=0$ corresponds to the transition from LOC to HOC (see X-axis in Fig. Figure 2B).

\section{Oscillation p-Values}

For calculation of oscillation $\mathrm{p}$-values $p_{\mathrm{DFT}}$ on read-count level the time series were permutated $N_{p}=10,000$ times, and random amplitude $\tilde{A}_{2}$ calculated. The $\mathrm{p}$-value was estimated as the fraction of permutations for which the random amplitude was larger than the observed amplitude $A_{2}$ (eqn. 2). This analysis was performed with the script genome0scillation. $\mathrm{R}$ from the segmenTools git repository. Oscillation $\mathrm{p}$-values $p_{\text {rain }}$ on segment level were calculated with the R package rain (Thaben and Westermark, 2014) using period $P=0.65 \mathrm{~h}$ and time step $\delta t=4 \mathrm{~min}$. This analysis was done with the script segmentDynamics. $\mathrm{R}$ from the segmenTools git repository. 


\section{Segmentation of RNA-seq Read-Counts}

The data were pre-segmented into expressed and weakly expressed chromosomal domains by a previously described heuristic (Machné et al., 2017) with a minor correction that splits presegments at chromosome ends. Pre-segmentation was done with the script presegment. $\mathrm{R}$ from the segmenTools script collection; Figure S7 provides pre-segment length distributions and run parameters. Pre-segments were then individually split into non-overlapping segments with coherent temporal expression profiles by the segmenTier algorithm, using optimized parameters from our previous study (Machné et al., 2017). Shortly, the arcsinh-transformed read-count data was Fouriertransformed (eq. 1); the first component $(k=0)$, reflecting the mean expression level, was arcsinhtransformed (eq. 4); and all other $(k>0)$ components were amplitude-scaled (eq. 3). The real and imaginary parts of the scaled DFT components $X_{k=0, ., 6}^{\prime}$ were then clustered into 12 groups with R's implementation of k-means (using the Hartigan-Wong method or if that failed, the MacQueen method). This clustering then provided the anchors for the similarity-based segmentation by the segmenTier, where we used the icor scoring function with exponent $\epsilon=2$, length penalty $M=150$, nuissance cluster penalty $M_{0}=100$, and nuissance cluster exponent $v=3$. This combination of parameters is achieved by arguments -trafo "ash" -dc.trafo "ash" -dft.range 1,2,3,4,5,6,7 -K 12 -Mn 100 -scores "icor" -scales 2 -M 150 -nui.cr 3 to the runSegmentier.R script in the segmenTools/scripts collection. All segments are provided in Dataset S2.

\section{Segment Clustering}

The means of read-counts covered by a segment were taken as segment time series. Periodic expression was analyzed by permutation analysis and DFT and by the R package rain. 11,248 segments with $p_{\text {rain }}<0.85$ were chosen for further analysis (Fig. S8E-F). The DFT of the segment time series was amplitude-scaled (eq. 3, Fig. S10A) and the first (constant) component $(k=0)$ was arcsinh-transformed (eq. 4). Real and imaginary parts of the scaled DFT components $X_{k=0, . .6}^{\prime}$ were then clustered with the flowClust algorithm (Lo et al., 2009) for cluster numbers $K=2, \ldots, 16$. The clustering with the maximal Bayesian Information Criterion, as reported by flowClust (Fig. S10B), was selected for further analysis. Clustering was performed by clusterTimeseries 2 function of segmenTools via the segmentDynamics. R script). The resulting clustering was sorted, re-labeled and colored automatically based on the means of their segments' expression phases (eq. 2). The clustering was further sub-divided into high-amplitude clusters enriched for coding genes and low-amplitude clusters (compare Fig. S11 and S12).

\section{Relative Protein Amplitudes}

3,174 segments overlapping with a coding region with Jaccard index $J>0.5$ and protein half-live $\left(\tau_{1 / 2}\right)$ annotation in (Christiano et al., 2014) were considered. The relative mRNA amplitudes were calculated from the DFT $X_{k}$ (eq. 1-2) of the first 19 time points (2 full cycles) of the RNA-seq read count time series as $A_{\mathrm{mRNA}}=X_{2} / X_{0}$, i.e., the ratio of the amplitudes of the $2^{\text {nd }}$ component $X_{2}$ ( 2 cycles) over the $0^{\text {th }}$ component $X_{0}$ (corresponds to the mean over all time points) of the DFT. Relative protein amplitudes $A_{\text {protein }}$ were then calculated with the analytical solution to an ordinary differential equation of rhythmic production, after equation S8 of (Lück et al., 2014), as

$$
A_{\text {protein }}=A_{\text {mRNA }} \frac{\gamma}{\sqrt{\gamma^{2}+\omega^{2}}},
$$

with oscillation frequency $\omega=\frac{1}{0.67} \mathrm{~h}^{-1}$ and total protein degradation rate $\gamma=\delta+\phi$, where the actual protein degradation rates $\delta$ were taken from (Christiano et al., 2014) and dilution by growth is the chemostat dilution rate $\phi=0.089 \mathrm{~h}^{-1}$. In this model, the relative amplitude $A_{\mathrm{mRNA}}$ is assumed to directly reflect periodic production,i.e., translational activity. Total amounts or translation rates are not required, and only a relative amplitude of protein amount can be calculated. Predicted protein amplitudes are provided in Dataset S3. 


\section{Peak Width Analysis}

For each high-amplitude segment $\left(p_{\text {rain }}<0.0001\right)$ the time series was interpolated to $1^{\circ}$ resolution $(0.105 \mathrm{~min})$, and the oscillation phase $\phi_{2}$ (eq. 2) was used to scan the times spent above the temporal median $\tilde{x}$ during the first $\left(C_{1}\right)$ and the second $\left(C_{2}\right)$ full cycle in the data set (horizontal arrows in Figure 2B). These times were recorded as the peak widths $W_{1}$ and $W_{2}$. The peak width change is the difference $\Delta W=W_{2}-W_{1}$, and $\Delta \Delta W$ was calculated as the difference of the medians of $\Delta W$ of two distinct segment clusters. See Figure S20A for details.

\section{Cluster and Segment Enrichment Analyses}

Segment Overlap Analysis: J-R Plots

To analyze overlaps between two distinct genome segmentations, a query set $\mathbf{Q}$ and a target set

T, we scanned each target segment for overlapping query segments, calculated Jaccard indices

$J=|Q \cap T| /|Q \cup T|$ and length ratios $R=|Q| /|T|$ for all overlapping pairs of segments:

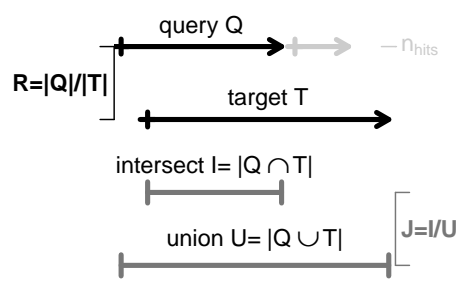

where $|x|$ denotes the length of a segment $x$. Only the query segment $Q_{i}$ with the highest $J_{i, m}$ for a given target $T_{m}$ was considered, resulting in $J$ and $R$ values for each target segment. Herein, we used our segmentation as the query set and various genome annotations and previously published transcript data sets as target sets.

While the distribution of $J$ values provides a measure how well query and target sets overlap overall, the distribution of length ratios $R$ additionally indicates systematic length differences. Plotting the 2D distributions of individual $J$ and $R$ values for overlapping query and target pairs then provides an overview of how two segmentations relate to each other (J-R plots). For symmetry, the J-R plots (Figure 2D, S9A-D) are mirrored at $R=1$, such that $R$ is plotted for $R<1$ and $\frac{1}{R}$ is plotted for $R>1$. Enrichment at $R<1$ then indicates that the target set tends to contain longer segments than the query set, and vice versa. For example, when comparing our segments (query) with annotated Open Reading Frames (target), the enrichment of values close to $J=1$ and at $R>0$ (Figure 2D) indicates that, as expected, transcribed segments tend to be longer than the ORF they cover. J-R plots are implemented in the jrplot function of the segmenTools package.

\section{Segment Overlap Enrichment Tests}

Global enrichments of overlaps between two distinct sets of genomic intervals (Figure 4B-D and S22) were calculated by a segment permutation analysis with $n=1000$ permutations, and counting the number of permutations $p_{h}$ which reached the same or higher total Jaccard Index $J_{\text {tot }}=\frac{I}{U}$ between all segments of the query and target sets and calculated the $\mathrm{p}$-value $p=p_{h} / n$. Specifically, the function segment Jaccard of the segmenTools package analyses multiple segment classes (e.g. co-expression cohort clusters) in both, query and target set, in one step. For the permutation test, the order of all query segments and inter-segments is permutated, which conserves the length distribution. $J$ and $p$ are calculated for each combination of segment classes in the query and target sets. The total counts of overlapping pairs and p-values are stored ("enrichment tables") for informative plots ("Enrichment Profiles"). 


\section{Cluster-Cluster Enrichment Tests}

Categorical enrichments, e.g. coding gene co-expression cohorts vs. gene annotations, were analyzed by cumulative hypergeometric distribution tests (R's phyper) using segmenTools's clusterCluster function and the clusterAnnotation wrapper for $\mathrm{GO}$ and and protein complex analysis, which compares overlaps of each pair of two distinct classifications into multiple classes, and stores overlap counts and p-values ("enrichment tables") for informative plots (see "Enrichment Profiles").

We considered segments that overlapped with an ORF with a Jaccard index $J>0.5$ in gene-wise cluster enrichment analyses, except for the analysis of protein complex analysis, where all segments that overlap with a gene $J>0$, and the one with maximal $J$ if several segments overlap, were considered to comprehensively capture complex co-expression and differential expression.

\section{Enrichment Profiles}

The results of multi-class enrichment tests (segment overlaps or cluster-cluster categorical overlaps) were visualized as colored table plots, e.g. Figure 3B), using segmenTools' function plot0verlaps. The total counts of overlapping pairs are plotted as text, where the text color is selected based on a p-value cutoff $p_{\text {txt }}$ (as indicated). The background color gray level of each field scales with $\log _{2}(p)$, such that fields with a minimal p-value $p_{\min }$ (as indicated) are black.

For intuitively informative plots the enrichment tables (see Cluster-Cluster Enrichment Tests) were sorted. Table rows were sorted along the other dimension (table columns) such that all categories enriched above a certain threshold $p_{\text {sort }}$ in the first column cluster are moved to the top, and, within, sorted by increasing p-values. Next, the same sorting is applied to all remaining row clusters for the second column cluster, and so on until the last column cluster. Remaining row clusters are either plotted unsorted below a red line or removed. This is especially useful to visualize enrichment of functional categories along the temporal program of co-expression cohorts, e.g., Figure 3B and E. This sorting is implemented in segmenTools' function sortOverlaps.

\section{Supporting Data}

\section{Dataset S1:}

Reactor data, including all calculated rates and RNA-seq samples IDs.

\section{Dataset S2:}

All 36,928 segments reported by segmenTier, incl. genome coordinates, cluster labels, read-counts, oscillation values (amplitude $A_{2}$, phase $\phi_{2}$, p-value $p_{\text {rain }}$ ), coding gene and SUT overlaps, and all time points, using the sampling IDs (2-25) indicated in the reactor data.

\section{Dataset S3:}

Predicted relative protein amplitudes for 3,174 coding genes that overlap with a segment with $J>0$ and for which protein half-life data was available in (Christiano et al., 2014), incl. segment ID (as in Dataset S1), the calculated relative RNA amplitudes, the cluster assignment of the segment, and GO terms associated with the protein.

\section{Author Contributions}

DBM designed and performed the experiment, SHB performed the RNA-seq read mapping, RM and PFS developed the segmentation algorithm. RM and IMA analyzed the time series data. All authors contributed to data interpretation and to writing of the manuscript.

\section{Acknowledgments}

We thank Sarah Lück, Oliver Ebenhöh, Ovidiu Popa, Chilperic Armel Foko Kuate and St. Elmo Wilken for inspiring discussions of the data and critical review of the manuscript. 


\section{Funding}

DBM was funded by a partnering award from Japan Science and Technology Agency, Yamagata prefectural government and the City of Tsuruoka. RM was funded by the Deutsche Forschungsgemeinschaft, grants AX 84/4-1 and STA 850/30-1.

\section{References}

Aguilera J, Van Dijken JP, De Winde JH, Pronk JT. Carbonic anhydrase (Nce103p): an essential biosynthetic enzyme for growth of Saccharomyces cerevisiae at atmospheric carbon dioxide pressure. Biochem J. 2005 Oct; 391(Pt 2):311-316.

Amariei C, Machné R, Stolc V, Soga T, Tomita M, Murray DB. Time resolved DNA occupancy dynamics during the respiratory oscillation uncover a global reset point in the yeast growth program. Microb Cell. 2014 Sep; 1(9):279-288. doi: 10.15698/mic2014.09.166.

Aon MA, Roussel MR, Cortassa S, O'Rourke B, Murray DB, Beckmann M, Lloyd D. The scale-free dynamics of eukaryotic cells. PLoS One. 2008; 3(11):e3624. doi: 10.1371/journal.pone.0003624.

Balakrishnan R, Park J, Karra K, Hitz BC, Binkley G, Hong EL, Sullivan J, Micklem G, Cherry JM. YeastMine-an integrated data warehouse for Saccharomyces cerevisiae data as a multipurpose tool-kit. Database (Oxford). 2012; 2012:bar062. doi: 10.1093/database/bar062.

Belle A, Tanay A, Bitincka L, Shamir R, O'Shea EK. Quantification of protein half-lives in the budding yeast proteome. Proc Natl Acad Sci U S A. 2006 Aug; 103(35):13004-13009. doi: 10.1073/pnas.0605420103.

Bellgardt KH. Analysis of synchronous growth of baker's yeast. Part I: Development of a theoretical model for sustained oscillations. Journal of Biotechnology. 1994; 35(1):19 - 33. doi: 10.1016/0168-1656(94)90187-2.

Bellgardt KH. Analysis of synchronous growth of baker's yeast. Part II: Comparison of model prediction and experimental data. Journal of Biotechnology. 1994; 35(1):35 - 49. doi: 10.1016/0168-1656(94)90188-0.

Beuse M, Bartling R, Kopmann A, Diekmann H, Thoma M. Effect of the dilution rate on the mode of oscillation in continuous cultures of Saccharomyces cerevisiae. J Biotechnol. 1998 Mar; 61(1):15-31.

Bleykasten-Grosshans C, Friedrich A, Schacherer J. Genome-wide analysis of intraspecific transposon diversity in yeast. BMC Genomics. 2013 Jun; 14:399.

Brauer MJ, Saldanha AJ, Dolinski K, Botstein D. Homeostatic adjustment and metabolic remodeling in glucoselimited yeast cultures. Mol Biol Cell. 2005 May; 16(5):2503-2517. doi: 10.1091/mbc.E04-11-0968.

Bristow SL, Leman AR, Simmons Kovacs LA, Deckard A, Harer J, Haase SB. Checkpoints couple transcription network oscillator dynamics to cell-cycle progression. Genome Biol. 2014; 15(9):446. doi: 10.1186/s13059014-0446-7.

Bryan AK, Goranov A, Amon A, Manalis SR. Measurement of mass, density, and volume during the cell cycle of yeast. Proc Natl Acad Sci U S A. 2010 Jan; 107(3):999-1004.

Burnetti AJ, Aydin M, Buchler NE. Cell cycle Start is coupled to entry into the yeast metabolic cycle across diverse strains and growth rates. Mol Biol Cell. 2016 Jan; 27(1):64-74. doi: 10.1091/mbc.E15-07-0454.

Causton HC, Feeney KA, Ziegler CA, O'Neill JS. Metabolic Cycles in Yeast Share Features Conserved among Circadian Rhythms. Curr Biol. 2015 Apr; 25(8):1056-1062. doi: 10.1016/j.cub.2015.02.035.

Chin SL, Marcus IM, Klevecz RR, Li CM. Dynamics of oscillatory phenotypes in Saccharomyces cerevisiae reveal a network of genome-wide transcriptional oscillators. FEBS J. 2012 Mar; 279(6):1119-1130. doi: 10.1111/j.17424658.2012.08508.x.

Christiano R, Nagaraj N, Frohlich F, Walther TC. Global proteome turnover analyses of the Yeasts S. cerevisiae and S. pombe. Cell Rep. 2014 Dec; 9(5):1959-1965. doi: 10.1016/j.celrep.2014.10.065.

Curcio MJ, Lutz S, Lesage P. The Ty1 LTR-retrotransposon of budding yeast, Saccharomyces cerevisiae. Microbiol Spectr. 2015 Apr; 3(2):1-35.

van Dijk EL, Chen CL, d'Aubenton Carafa Y, Gourvennec S, Kwapisz M, Roche V, Bertrand C, Silvain M, Legoix-Ne P, Loeillet S, Nicolas A, Thermes C, Morillon A. XUTs are a class of Xrn1-sensitive antisense regulatory non-coding RNA in yeast. Nature. 2011 Jul; 475(7354):114-117. doi: 10.1038/nature10118. 
Duboc P, von Stockar U. Modeling of oscillating cultivations of Saccharomyces cerevisiae: Identification of population structure and expansion kinetics based on on-line measurements. Chemical Engineering Science. 2000; 55(1):149-160. doi: 10.1016/S0009-2509(99)00301-2.

Ellison MA, Lederer AR, Warner MH, Mavrich TN, Raupach EA, Heisler LE, Nislow C, Lee MT, Arndt KM. The Paf1 Complex Broadly Impacts the Transcriptome of Saccharomyces cerevisiae. Genetics. 2019 Jul; 212(3):711-728.

Feltham J, Xi S, Murray S, Wouters M, Urdiain-Arraiza J, George C, Townley A, Roberts E, Fisher R, Liberatori S, Mohammed S, Kessler B, Mellor J. Transcriptional changes are regulated by metabolic pathway dynamics but decoupled from protein levels. bioRxiv. 2019; doi: 10.1101/833921.

Futcher B. Metabolic cycle, cell cycle, and the finishing kick to Start. Genome Biol. 2006; 7(4):107. doi: 10.1186/gb-2006-7-4-107.

Gasch AP, Spellman PT, Kao CM, Carmel-Harel O, Eisen MB, Storz G, Botstein D, Brown PO. Genomic expression programs in the response of yeast cells to environmental changes. Mol Biol Cell. 2000 Dec; 11(12):4241-4257.

Hani J, Feldmann H. tRNA genes and retroelements in the yeast genome. Nucleic Acids Res. 1998 Feb; 26(3):689-696.

Heinzle E. Mass spectrometry for on-line monitoring of biotechnological processes. In: Biotechnology Methods Springer; 1987.p. 1-45.

Heinzle E, Dunn IJ, Furukawa K, Tanner RD. Modelling of sustained oscillations observed in continuous culture of Saccharomyces cerevisiae. In: Halme A, editor. Modelling and control of biotechnical processes International Federation of Automatic Control, Helsinki, Finland: Pergamon Press; 1983. p. 57-65.

Hjortso MA, Nielsen J. Population balance models of autonomous microbial oscillations. J Biotechnol. 1995 Oct; 42(3):255-269.

Hoffmann S, Otto C, Doose G, Tanzer A, Langenberger D, Christ S, Kunz M, Holdt LM, Teupser D, Hackermüller J, Stadler PF. A multi-split mapping algorithm for circular RNA, splicing, trans-splicing and fusion detection. Genome Biol. 2014; 15(2):R34. doi: 10.1186/gb-2014-15-2-r34.

Jorgensen P, Rupes I, Sharom JR, Schneper L, Broach JR, Tyers M. A dynamic transcriptional network communicates growth potential to ribosome synthesis and critical cell size. Genes Dev. 2004 Oct; 18(20):2491-2505. doi: 10.1101/gad.1228804.

Keulers M, Kuriyama H. Extracellular Signaling in an Oscillatory Yeast Culture. In: Holcombe M, Paton R, editors. Information Processing in Cells and Tissues Springer US; 1998.p. 85-94. doi: 10.1007/978-1-4615-5345-8_10.

Keulers M, Satroutdinov AD, Suzuki T, Kuriyama H. Synchronization affector of autonomous short-periodsustained oscillation of Saccharomyces cerevisiae. Yeast. 1996 Jun; 12(7):673-682. doi: 10.1002/(SICI)10970061(19960615)12:7<673::AID-YEA958>3.0.CO;2-C.

Klevecz RR, Bolen J, Forrest G, Murray DB. A genomewide oscillation in transcription gates DNA replication and cell cycle. Proc Natl Acad Sci U S A. 2004 Feb 3; 101(5):1200-5. doi: 10.1073/pnas.0306490101.

Kuang Z, Cai L, Zhang X, Ji H, Tu BP, Boeke JD. High-temporal-resolution view of transcription and chromatin states across distinct metabolic states in budding yeast. Nat Struct Mol Biol. 2014 Oct; 21(10):854-863. doi: 10.1038/nsmb.2881.

Küenzi MT, Fiechter A. Changes in carbohydrate composition and trehalase-activity during the budding cycle of Saccharomyces cerevisiae. Archives of Microbiology. 1969; 64(4):396-407. doi: 10.1007/BF00417021, 10.1007/BF00417021.

Langdon EM, Qiu Y, Ghanbari Niaki A, McLaughlin GA, Weidmann CA, Gerbich TM, Smith JA, Crutchley JM, Termini CM, Weeks KM, Myong S, Gladfelter AS. mRNA structure determines specificity of a polyQ-driven phase separation. Science. 2018 May; 360(6391):922-927. doi: 10.1126/science.aar7432.

Levin JZ, Yassour M, Adiconis X, Nusbaum C, Thompson DA, Friedman N, Gnirke A, Regev A. Comprehensive comparative analysis of strand-specific RNA sequencing methods. Nat Methods. 2010 Sep; 7(9):709-715. doi: 10.1038/nmeth.1491.

Li CM, Klevecz RR. A rapid genome-scale response of the transcriptional oscillator to perturbation reveals a period-doubling path to phenotypic change. Proc Natl Acad Sci U S A. 2006 Oct 31; 103(44):16254-9. 
Lindner P. Mikroskopische Betriebskontrolle in den Gärungsgewerben: mit einer Einführung in die Hefenreinkultur, Infektionslehre und Hefenkunde, für Studierende und Praktiker bearbeitet. P. Parey; 1895. https://books.google.de/books?id=1VFIAAAAYAAJ.

Lindner P. Das Biosproblem in der Hefeforschung. Berichte der Deutschen Botanischen Gesellschaft. 1919; 37(11):34-40. doi: 10.1111/j.1438-8677.1919.tb07801.x.

Liu X, Oh S, Peshkin L, Kirschner MW. Computationally enhanced quantitative phase microscopy reveals autonomous oscillations in mammalian cell growth. Proc Natl Acad Sci U S A. 2020 Oct; doi: 10.1073/pnas.2002152117.

Lloyd D, Murray DB. Redox rhythmicity: clocks at the core of temporal coherence. Bioessays. 2007 May; 29(5):465-473. doi: 10.1002/bies.20575.

Lo K, Hahne F, Brinkman RR, Gottardo R. flowClust: a Bioconductor package for automated gating of flow cytometry data. BMC Bioinformatics. 2009; 10:145. doi: 10.1186/1471-2105-10-145.

Lück S, Thurley K, Thaben PF, Westermark PO. Rhythmic degradation explains and unifies circadian transcriptome and proteome data. Cell Rep. 2014 Oct; 9(2):741-751.

Maaløe O. Regulation of the Protein-Synthesizing Machinery-Ribosomes, tRNA, Factors, and So On. In: Goldberger RF, editor. Biological Regulation and Development: Gene Expression Boston, MA: Springer US; 1979.p. 487-542. doi: 10.1007/978-1-4684-3417-0_12.

Machné R. Temporal Organization of Growth in Saccharomyces cerevisiae. PhD thesis, Theoretical Biochemistry Group, University of Vienna; 2017.

Machné R, Murray DB. The yin and yang of yeast transcription: elements of a global feedback system between metabolism and chromatin. PLoS One. 2012; 7(6):e37906. doi: 10.1371/journal.pone.0037906.

Machné R, Murray DB, Stadler PF. Similarity-Based Segmentation of Multi-Dimensional Signals. Sci Rep. 2017 Sep; 7(1):12355. doi: 10.1038/s41598-017-12401-8.

Mandl I, Grauer A, Neuberg C. Solubilization of insoluble matter in nature; I. The part played by salts of adenosinetriphosphate. Biochim Biophys Acta. 1952 Jun; 8(6):654-663.

Marison I, Liu JS, Ampuero S, Von Stockar U, Schenker B. Biological reaction calorimetry: development of high sensitivity bio-calorimeters. Thermochimica Acta. 1998; 309(1-2):157-173.

Martens JA, Laprade L, Winston F. Intergenic transcription is required to repress the Saccharomyces cerevisiae SER3 gene. Nature. 2004 Jun 3; 429(6991):571-4.

Martin R, Pohlers S, Muhlschlegel FA, Kurzai O. CO2 sensing in fungi: at the heart of metabolic signaling. Curr Genet. 2017 May; doi: 10.1007/s00294-017-0700-0.

McClintock B. The origin and behavior of mutable loci in maize. Proc Natl Acad Sci U S A. 1950 Jun; 36(6):344355.

Mellor J. The molecular basis of metabolic cycles and their relationship to circadian rhythms. Nat Struct Mol Biol. 2016 Dec; 23(12):1035-1044. doi: 10.1038/nsmb.3311.

von Meyenburg HK. Energetics of the budding cycle of Saccharomyces cerevisiae during glucose limited aerobic growth. Archives of Microbiology. 1969; 66:289-303. doi: 10.1007/BF00414585, 10.1007/BF00414585.

von Meyenburg K. Katabolit-Repression und der Sprossungszyklus von Saccharomyces cerevisiae. PhD thesis, ETH Zürich; 1969.

Molenaar D, van Berlo R, de Ridder D, Teusink B. Shifts in growth strategies reflect tradeoffs in cellular economics. Mol Syst Biol. 2009; 5:323. doi: 10.1038/msb.2009.82.

Moller HD, Larsen CE, Parsons L, Hansen AJ, Regenberg B, Mourier T. Formation of Extrachromosomal Circular DNA from Long Terminal Repeats of Retrotransposons in Saccharomyces cerevisiae. G3 (Bethesda). 2015 Dec; 6(2):453-462.

Moreno-Risueno MA, Benfey PN. Time-based patterning in development: The role of oscillating gene expression. Transcription. 2011 May; 2(3):124-129. doi: 10.4161/trns.2.3.15637. 
Müller D. Model-Assisted Analysis of Cyclic AMP Signal Transduction in Saccharomyces cerevisiae — cAMP as Dynamic Coordinator of Energy Metabolism and Cell Cycle Progression. PhD thesis, Universität Stuttgart; 2006.

Münch T, Sonnleitner B, Fiechter A. The decisive role of the Saccharomyces cerevisiae cell cycle behaviour for dynamic growth characterization. J Biotechnol. 1992 Feb; 22(3):329-351.

Murray DB, Beckmann M, Kitano H. Regulation of yeast oscillatory dynamics. Proc Natl Acad Sci U S A. 2007 Feb; 104(7):2241-2246. doi: 10.1073/pnas.0606677104.

Murray DB, Klevecz RR, Lloyd D. Generation and maintenance of synchrony in Saccharomyces cerevisiae continuous culture. Exp Cell Res. 2003 Jul 1; 287(1):10-5.

Nguyen T, Fischl H, Howe FS, Woloszczuk R, Serra Barros A, Xu Z, Brown D, Murray SC, Haenni S, Halstead JM, O'Connor L, Shipkovenska G, Steinmetz LM, Mellor J. Transcription mediated insulation and interference direct gene cluster expression switches. Elife. 2014; 3:e03635. doi: 10.7554/eLife.03635.

Niessing D, Jansen RP, Pohlmann T, Feldbrugge M. mRNA transport in fungal top models. Wiley Interdiscip Rev RNA. 2018 Jan; 9(1).

Nocetti N, Whitehouse I. Nucleosome repositioning underlies dynamic gene expression. Genes Dev. 2016 Mar; 30(6):660-672. doi: 10.1101/gad.274910.115.

O' Neill JS, Hoyle NP, Robertson JB, Edgar RS, Beale AD, Peak-Chew SY, Day J, Costa ASH, Frezza C, Causton HC. Eukaryotic cell biology is temporally coordinated to support the energetic demands of protein homeostasis. Nat Commun. 2020 Sep; 11(1):4706. doi: 10.1038/s41467-020-18330-x.

O’Duibhir E, Lijnzaad P, Benschop JJ, Lenstra TL, van Leenen D, Groot Koerkamp MJ, Margaritis T, Brok MO, Kemmeren P, Holstege FC. Cell cycle population effects in perturbation studies. Mol Syst Biol. 2014 Jun; 10:732. doi: 10.15252/msb.20145172.

Orlando DA, Lin CY, Bernard A, Wang JY, Socolar JE, Iversen ES, Hartemink AJ, Haase SB. Global control of cell-cycle transcription by coupled CDK and network oscillators. Nature. 2008 Jun; 453(7197):944-947. doi: 10.1038/nature06955.

Palkova Z, Devaux F, Icicova M, Minarikova L, Le Crom S, Jacq C. Ammonia pulses and metabolic oscillations guide yeast colony development. Mol Biol Cell. 2002 Nov; 13(11):3901-3914. doi: 10.1091/mbc.E01-12-0149.

Parkhomchuk D, Borodina T, Amstislavskiy V, Banaru M, Hallen L, Krobitsch S, Lehrach H, Soldatov A. Transcriptome analysis by strand-specific sequencing of complementary DNA. Nucleic Acids Res. 2009 Oct; 37(18):e123. doi: 10.1093/nar/gkp596.

Patel A, Malinovska L, Saha S, Wang J, Alberti S, Krishnan Y, Hyman AA. ATP as a biological hydrotrope. Science. 2017 May; 356(6339):753-756. doi: 10.1126/science.aaf6846.

Porro D, Martegani E, Ranzi BM, Alberghina L. Oscillations in continuous cultures of budding yeast: a segregated parameter analysis. Biotechnol Bioeng. 1988 Aug; 32(4):411-417. doi: 10.1002/bit.260320402.

Pu S, Wong J, Turner B, Cho E, Wodak SJ. Up-to-date catalogues of yeast protein complexes. Nucleic Acids Res. 2009 Feb; 37(3):825-831. doi: 10.1093/nar/gkn1005.

Sasidharan K, Amariei C, Tomita M, Murray DB. Rapid DNA, RNA and protein extraction protocols optimized for slow continuously growing yeast cultures. Yeast. 2012 Aug; 29(8):311-322. doi: 10.1002/yea.2911.

Sasidharan K, Tomita M, Aon M, Lloyd D, Murray DB. Time-Structure of the Yeast Metabolism In vivo. In: Goryanin II, Goryachev AB, editors. Advances in Systems Biology, vol. 736 of Advances in Experimental Medicine and Biology Springer New York; 2012.p. 359-379. doi: 10.1007/978-1-4419-7210-1_21.

Satroutdinov AD, Kuriyama H, Kobayashi H. Oscillatory metabolism of Saccharomyces cerevisiae in continuous culture. FEMS Microbiol Lett. 1992 Nov; 77(1-3):261-267. doi: 10.1016/0378-1097(92)90167-m.

Schwarz A, Beck M. The Benefits of Cotranslational Assembly: A Structural Perspective. Trends Cell Biol. 2019 Aug; doi: 10.1016/j.tcb.2019.07.006.

Servant G, Pinson B, Tchalikian-Cosson A, Coulpier F, Lemoine S, Pennetier C, Bridier-Nahmias A, Todeschini $A L$, Fayol H, Daignan-Fornier B, Lesage $P$. Tye7 regulates yeast Ty1 retrotransposon sense and antisense transcription in response to adenylic nucleotides stress. Nucleic Acids Res. 2012 Jul; 40(12):5271-5282. doi: 10.1093/nar/gks166. 
Shiber A, Doring K, Friedrich U, Klann K, Merker D, Zedan M, Tippmann F, Kramer G, Bukau B. Cotranslational assembly of protein complexes in eukaryotes revealed by ribosome profiling. Nature. 2018 Sep; 561(7722):268272. doi: 10.1038/s41586-018-0462-y.

Slavov N, Botstein D. Coupling among growth rate response, metabolic cycle, and cell division cycle in yeast. Mol Biol Cell. 2011 Jun; 22(12):1997-2009. doi: 10.1091/mbc.E11-02-0132.

Slavov N, Botstein D, Caudy A. Extensive regulation of metabolism and growth during the cell division cycle. ArXiv e-prints. 2014 Jun; arXiv:1406.3398.

Slavov N, Macinskas J, Caudy A, Botstein D. Metabolic cycling without cell division cycling in respiring yeast. Proc Natl Acad Sci U S A. 2011 Nov; 108(47):19090-19095. doi: 10.1073/pnas.1116998108.

Sohn HY, Murray DB, Kuriyama H. Ultradian oscillation of Saccharomyces cerevisiae during aerobic continuous culture: hydrogen sulphide mediates population synchrony. Yeast. 2000 Sep 30; 16(13):1185-90. doi: 10.1002/1097-0061(20000930)16:13<1185::AID-YEA619>3.0.CO;2-W.

Sonnleitner B, Käppeli O. Growth of Saccharomyces cerevisiae is controlled by its limited respiratory capacity: Formulation and verification of a hypothesis. Biotechnol Bioeng. 1986 Jun; 28(6):927-937. doi: 10.1002/bit.260280620.

Sonnleitner B, Rothen SA, Kuriyama H. Dynamics of glucose consumption in yeast. Biotechnol Prog. 1997 Jan; 13(1):8-13. doi: 10.1021/bp960094+.

Strässle C, Sonnleitner B, Fiechter A. A predictive model for the spontaneous synchronization of Saccharomyces cerevisiae grown in continuous culture. I. Concept. Journal of Biotechnology. 1988; 7(4):299 - 317 . doi: 10.1016/0168-1656(88)90042-9.

Thaben PF, Westermark PO. Detecting rhythms in time series with RAIN. J Biol Rhythms. 2014 Dec; 29(6):391-400. doi: $10.1177 / 0748730414553029$.

Thompson DM, Parker R. Cytoplasmic decay of intergenic transcripts in Saccharomyces cerevisiae. Mol Cell Biol. 2007 Jan; 27(1):92-101. doi: 10.1128/MCB.01023-06.

Tu BP, Kudlicki A, Rowicka M, McKnight SL. Logic of the yeast metabolic cycle: temporal compartmentalization of cellular processes. Science. 2005 Nov 18; 310(5751):1152-8.

Verduyn C, Stouthamer AH, Scheffers WA, van Dijken JP. A theoretical evaluation of growth yields of yeasts. Antonie Van Leeuwenhoek. 1991 Jan; 59(1):49-63.

Wang GZ, Hickey SL, Shi L, Huang HC, Nakashe P, Koike N, Tu BP, Takahashi JS, Konopka G. Cycling Transcriptional Networks Optimize Energy Utilization on a Genome Scale. Cell Rep. 2015 Dec; 13(9):1868-1880. doi: 10.1016/j.celrep.2015.10.043.

Wolf J, Sohn H, Heinrich R, Kuriyama H. Mathematical analysis of a mechanism for autonomous metabolic oscillations in continuous culture of Saccharomyces cerevisiae. FEBS Lett. 2001 Jun 22; 499(3):230-4.

Xu Z, Wei W, Gagneur J, Perocchi F, Clauder-Munster S, Camblong J, Guffanti E, Stutz F, Huber W, Steinmetz LM. Bidirectional promoters generate pervasive transcription in yeast. Nature. 2009 Feb; 457(7232):1033-1037. doi: 10.1038/nature07728.

Xu Z, Yaguchi S, Tsurugi K. Gts1p stabilizes oscillations in energy metabolism by activating the transcription of TPS1 encoding trehalose-6-phosphate synthase 1 in the yeast Saccharomyces cerevisiae. Biochem J. 2004 Oct; 383(Pt 1):171-178. doi: 10.1042/BJ20040967.

Yassour M, Pfiffner J, Levin JZ, Adiconis X, Gnirke A, Nusbaum C, Thompson DA, Friedman N, Regev A. Strandspecific RNA sequencing reveals extensive regulated long antisense transcripts that are conserved across yeast species. Genome Biol. 2010; 11(8):R87. doi: 10.1186/gb-2010-11-8-r87. 